Engineer Research and

Development Center

\title{
Rutting Performance of Cold-Applied Asphalt Repair Materials for Airfield Pavements
}

Ben C. Cox, John F. Rushing, and Web Floyd

June 2017

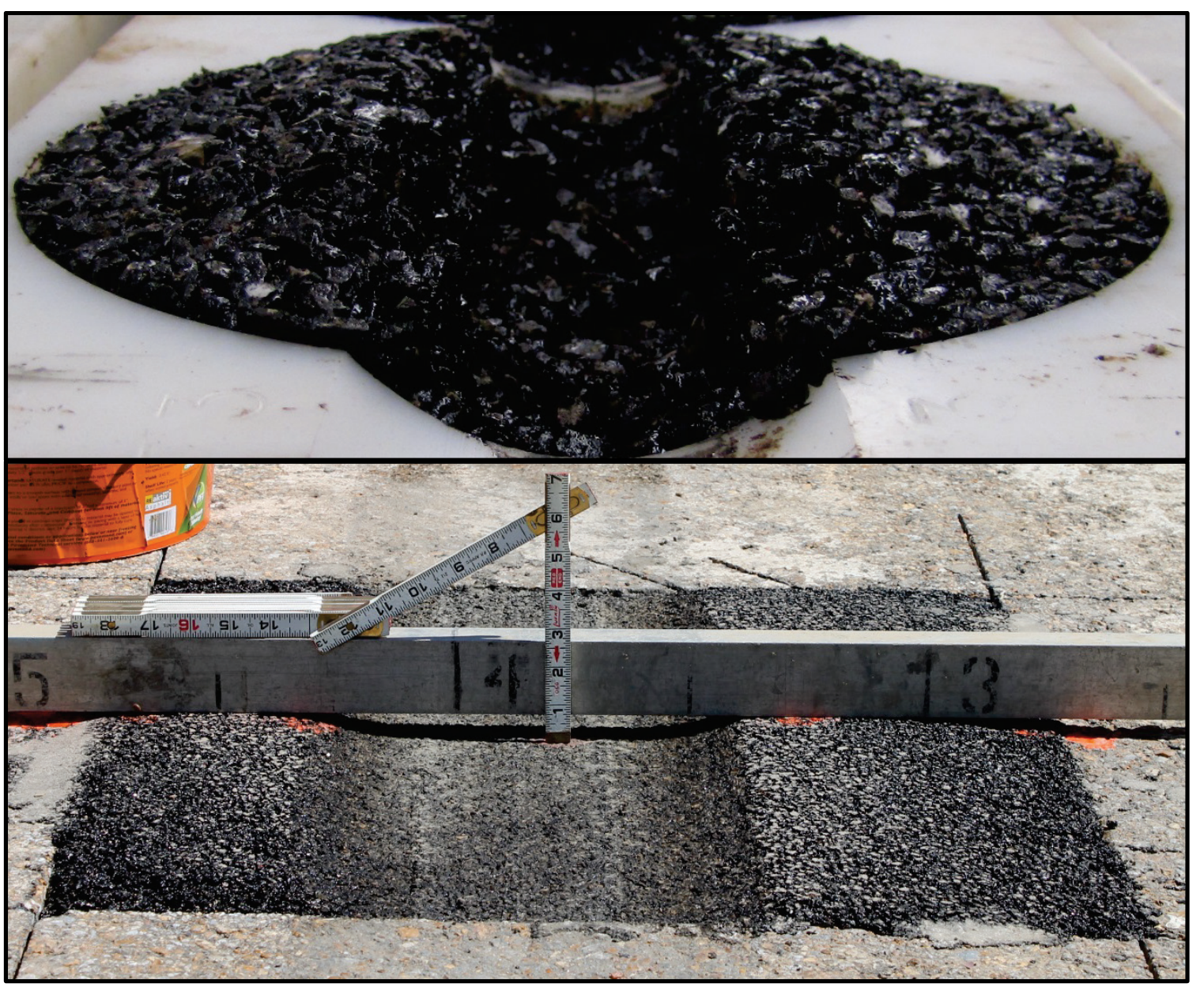


The U.S. Army Engineer Research and Development Center (ERDC) solves the nation's toughest engineering and environmental challenges. ERDC develops innovative solutions in civil and military engineering, geospatial sciences, water resources, and environmental sciences for the Army, the Department of Defense, civilian agencies, and our nation's public good. Find out more at www.erdc.usace.army.mil.

To search for other technical reports published by ERDC, visit the ERDC online library at http://acwc.sdp.sirsi.net/client/default. 


\section{Rutting Performance of Cold-Applied Asphalt Repair Materials for Airfield Pavements}

Ben C. Cox, John F. Rushing, and Web Floyd

Geotechnical and Structures Laboratory

U.S. Army Engineer Research and Development Center

3909 Halls Ferry Road

Vicksburg, MS 39180-6199

Final report

Approved for public release; distribution is unlimited.

Prepared for Headquarters, Air Force Civil Engineer Center

139 Barnes Avenue, Suite 1

Tyndall AFB, FL 32403-5319

Under Project 448464; Airfield Damage Repair (ADR) Modernization Program 


\section{Abstract}

Cold-applied asphalt mixtures are often used for pavement repair, primarily because of the small quantities involved and/or the unavailability of hotmixed asphalt. These cold-applied mixtures have poorer rutting resistance than hot mix asphalt because additives, often solvent, are required to provide adequate workability for them to be placed at ambient temperatures. This study primarily evaluated the rutting performance of nine commercial cold mix asphalt repair materials. Both laboratory wheel tracking data and field rutting performance were used in the evaluation with the focus on identifying suitable materials for repairing airfield pavements subjected to high-tire-pressure aircraft traffic. The commercial products included traditional cutback cold mixes as well as water-activated repair materials. Results from both laboratory and field rutting measurements showed that the water-activated materials outperform the products containing solvent. However, none of the commercial products achieved the target threshold of less than 1 in. rutting after 100 passes of simulated F-15E aircraft traffic. The laboratory wheel-tracking measurements proved to be a good indicator of field performance.

DISCLAIMER: The contents of this report are not to be used for advertising, publication, or promotional purposes. Citation of trade names does not constitute an official endorsement or approval of the use of such commercial products. All product names and trademarks cited are the property of their respective owners. The findings of this report are not to be construed as an official Department of the Army position unless so designated by other authorized documents. 


\section{Contents}
Abstract ii
Figures and Tables....................................................................................................................

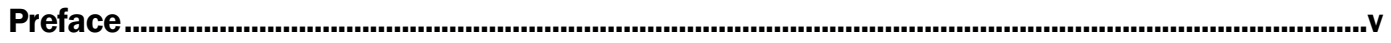

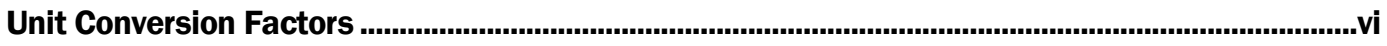

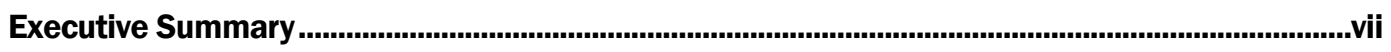

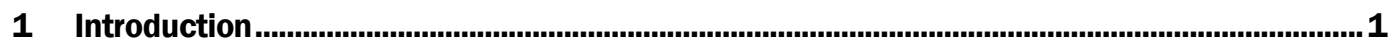

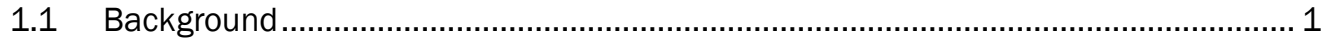

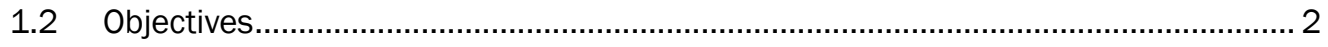

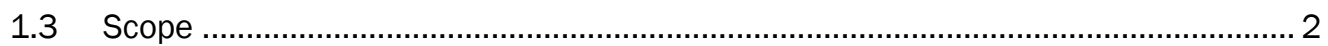

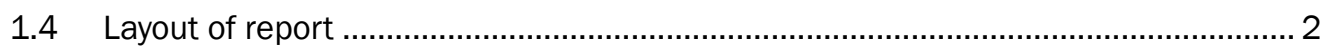

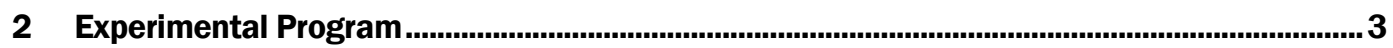

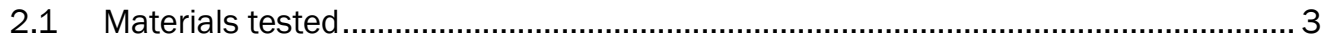

2.1.1 Cold mix asphalt materials .............................................................................. 3

2.1.2 Hot mix asphalt materials.............................................................................. 5

2.2 Laboratory test methods ................................................................................ 7

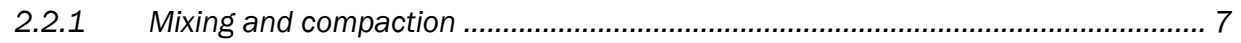

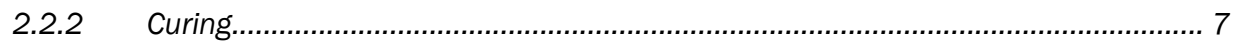

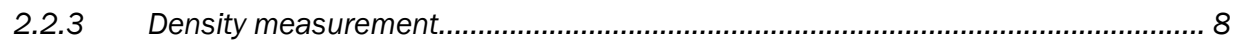

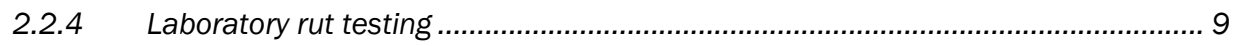

2.2.5 Indirect tensile strength testing ................................................................... 11

$2.3 \quad$ Field test methods .................................................................................... 12

2.3.1 Phase 1 field testing ................................................................................... 12

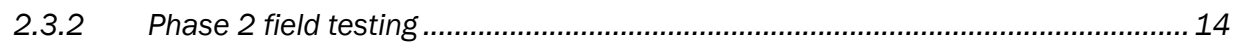

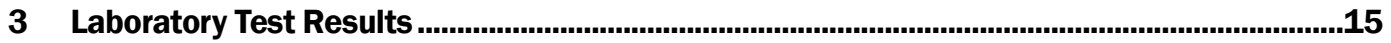

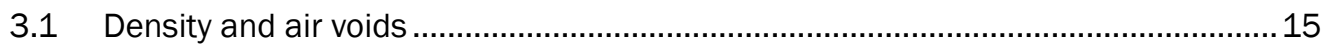

3.2 APA rutting...................................................................................................... 16

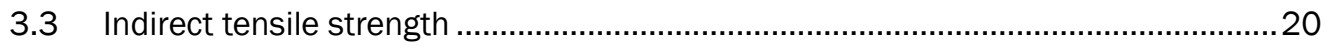

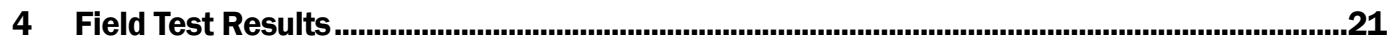

4.1 Phase 1 field testing...................................................................................... 21

4.2 Phase 2 field testing...................................................................................... 22

5 Discussion of Results ....................................................................................................25

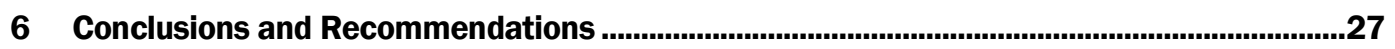

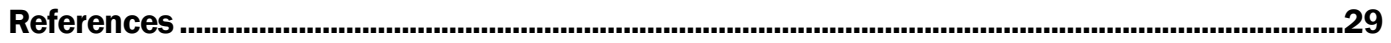

\section{Report Documentation Page}




\section{Figures and Tables}

\section{Figures}

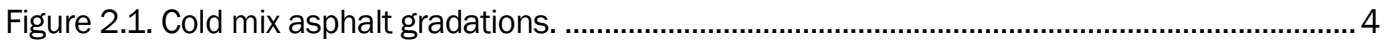

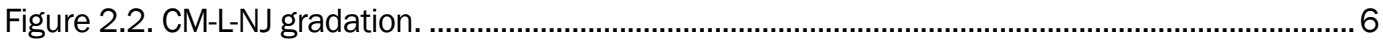

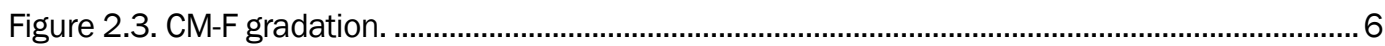

Figure 2.4. Photographs of laboratory mixing and compaction: (a) automatic Marshall compactor, (b) HMA bucket mixer, (c) Marshall mold filled with mix, (d) specimens on lab

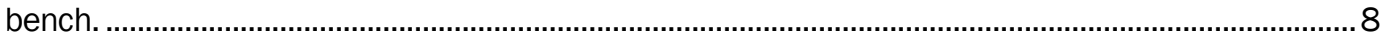

Figure 2.5. Typical cold mix specimen deformed by CoreLok bag. ……….........................................

Figure 2.6. Asphalt pavement analyzer: (a) overall, (b) inside of cabin. .............................................10

Figure 2.7. Typical tested APA specimen.......................................................................................11

Figure 2.8. Example field patch (IA shown): (a) before trafficking, (b) after trafficking.....................13

Figure 2.9. Heatwurx infrared heater. .......................................................................................13

Figure 2.10. F-15E load cart. .................................................................................................

Figure 3.1. Cold mix 1-day APA results. ..............................................................................................17

Figure 3.2. Cold mix 1 and 3 day APA results...........................................................................18

Figure 3.3. Cold mix 1, 3, and 5 day APA results. ...........................................................................18

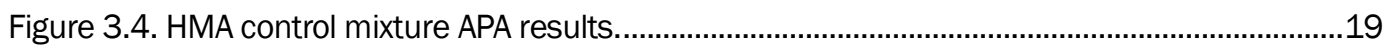

Figure 4.1. Phase 1 field rutting results by pass level......................................................................21

Figure 4.2. Phase 2 field rutting results by pass level. ...................................................................23

Figure 4.3. $\mathrm{CR}$ and $\mathrm{PC}$ comparison using straightedge rut measurements. ...................................23

Figure 4.4. Comparison of survey and straightedge rut measurements...........................................2

\section{Tables}

Table 2.1. Cold mix asphalt products evaluated. ..............................................................................

Table 2.2. Cold mix asphalt gradations by material. ………................................................................... 4

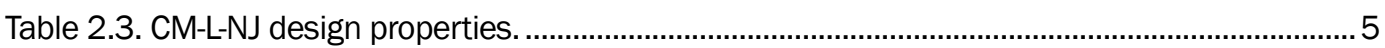

Table 2.4. APA test plan for cold mixes. .....................................................................................1

Table 3.1. Laboratory density and air void results.......................................................................15

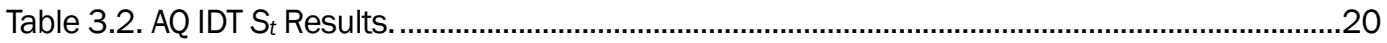




\section{Preface}

This study was conducted for the U.S. Air Force Civil Engineer Center (AFCEC), Tyndall Air Force Base, Florida. The technical manager for this project was Dr. Craig Rutland of AFCEC.

The work was performed by the Airfields and Pavements Branch (GMA) of the Engineering Systems and Materials Division (GM), U.S. Army Engineer Research and Development Center, Geotechnical and Structures Laboratory (ERDC-GSL).

At the time of publication, Dr. Timothy W. Rushing was Chief, CEERDGMA; Dr. Gordon W. McMahon was Chief, CEERD-GM; Dr. William P. Grogan was Deputy Director, ERDC-GSL; and Mr. Bartley P. Durst was Director.

COL Bryan S. Green was the Commander of ERDC, and Dr. David W. Pittman was the Director. 


\section{Unit Conversion Factors}

\begin{tabular}{|l|c|l|}
\hline Multiply & By & To Obtain \\
\hline degrees Fahrenheit & $(\mathrm{F}-32) / 1.8$ & degrees Celsius \\
\hline feet & 0.3048 & meters \\
\hline gallons (U.S. liquid) & $3.785412 \mathrm{E}-03$ & cubic meters \\
\hline inches & 0.0254 & meters \\
\hline miles per hour & 0.44704 & meters per second \\
\hline pounds (mass) & 0.45359237 & kilograms \\
\hline square feet & 0.09290304 & square meters \\
\hline square yards & 0.8361274 & square meters \\
\hline yards & 0.9144 & meters \\
\hline
\end{tabular}




\section{Executive Summary}

The U.S. Army Engineer Research and Development Center began a research project to study the performance of cold-applied asphalt mixtures for repairing airfield pavement. Laboratory air void, rutting, and indirect tensile strength data is presented in this report alongside field rutting data with simulated $\mathrm{F}-15 \mathrm{E}$ traffic for nine cold mix asphalt repair products and four conventional hot mix asphalt (HMA) control mixtures. Key observations from this study are as follows:

- In-place air void content levels of cold-applied mixtures were on the order of 2-3 times greater than typical target air void levels for HMA during construction. Inability to densify cold-applied asphalt mixtures is a major factor in poor rutting performance.

- Water-reactive cold mix products exhibited significantly more favorable rutting behaviors than traditional cutback materials used in previous investigations. The use of this category of materials has not been documented on airfield pavements.

- In field testing, rutting resistance of the HMA control mixture was on the order of 2-5 times greater than water-reactive cold mixes Aquaphalt ${ }^{\mathrm{TM}}$ 6.0 and Instant Asphalt ${ }^{\mathrm{TM}} 6.0$ (the only two products directly compared to HMA).

- The Asphalt Pavement Analyzer proved to be a useful tool in ranking product rutting performance on a laboratory-scale. Trends from laboratory testing matched field performance.

- Curing time meaningfully improved rutting results for cold-applied mixtures, particularly for the water-reactive products.

Based on findings in this study, key recommendations are as follows:

- Cure time should be investigated further for implementation into Tactics, Techniques, and Procedures (TTPs) to better optimize overall process expediency and rutting performance simultaneously.

- Lower-temperature testing should be conducted, particularly for the water-reactive products, to evaluate durability and cracking properties to ensure these materials are not prone to producing foreign object debris (FOD). 
- Further testing is recommended to determine if water-activated products could replace Instant Road Repair ${ }^{\circledR}$, the current recommended product, to provide improved early rutting performance. 


\section{Introduction}

\subsection{Background}

Cold-applied asphalt repair materials, commonly referred to as cold mixes, are often used for the rapid repair of airfield pavements, highway pavements, and municipal roads. Typically, these materials are utilized when plant-mixed hot mix asphalt (HMA) is not readily available or practical for use and because they can be placed with relative ease and speed. Speed of repair, specifically, is almost always a major concern for a typical rehabilitation project since it requires closure of the pavement to traffic for some period of time.

Logistical advantages such as material availability and speed of repair are usually accompanied with performance disadvantages. Cold mixes often exhibit rutting issues, and these could be significant when aircraft loadings are applied. With severe rutting, increased potential for foreign object debris (FOD) production is possible, posing a major risk to aircraft on airfield pavements.

In attempts to mitigate performance issues to the extent possible, MejíasSantiago et al. (2010) tested seven commercial-off-the-shelf (COTS) cold mix products to develop laboratory criteria to facilitate material selection in practice. All materials tested performed poorly, and, ultimately, cold mix repair products were not recommended for airfield pavement repairs subjected to high-tire-pressure aircraft such as the F-15E. Additionally, the suite of tests considered did not include any type of laboratory wheeltracking test.

This report builds off work conducted by Mejías-Santiago et al. (2010) in two notable ways. First, nine COTS cold mix products were tested in this study. Three of them were manufactured with water-reactive binder formulations, which were not considered in Mejías-Santiago et al. (2010). Second, laboratory wheel tracking of each material was assessed as an indicator of field rutting performance. 


\subsection{Objectives}

The main objectives of this study were as follows:

- Conduct laboratory testing on commercially available cold mix materials to characterize, among other properties, material rutting behavior,

- Conduct full-scale field repairs and subject them to simulated aircraft traffic,

- Evaluate results and relative merits of each cold mix product, particularly with respect to rutting performance.

\subsection{Scope}

This project consisted of a selection of nine available COTS cold patch asphalt repair materials identified by the research team under the guidance of the U.S. Air Force Civil Engineer Center. The selected materials were evaluated through laboratory tests and under controlled traffic conditions in the field to determine the ability of the repair materials to support F-15E aircraft traffic. Assessment of rutting performance was the focus of this report. Properties, such as air voids, were measured in some cases while others (e.g., durability, cracking, raveling) were not considered.

\subsection{Layout of report}

Chapter 2 details the experimental program including materials tested, laboratory testing, and field testing. Chapter 3 presents laboratory testing results, and Chapter 4 presents field testing results. Chapter 5 provides a discussion of the results, and Chapter 6 lists the conclusions and recommendations. 


\section{Experimental Program}

\subsection{Materials tested}

\subsubsection{Cold mix asphalt materials}

Table 2.1 lists the nine COTS cold mix asphalt materials tested in this study. Cold mix asphalt materials, further denoted cold mixes, were selected to reasonably represent available products on the market and were either cutback asphalt formulations or water-reactive formulations. Cutback asphalts generally refer to a combination of asphalt binder and petroleum solvent (e.g., diesel, kerosene). The primary function of the petroleum solvent in the system is to reduce viscosity and increase workability at "cold" (i.e., ambient) temperatures. Cutback formulations cure by volatilization of the solvent. Water-reactive formulations typically contain plant-based oils and a reaction-assisting material. When mixed with water, the materials chemically react and promote strength development.

Table 2.1. Cold mix asphalt products evaluated.

\begin{tabular}{|c|c|c|c|}
\hline ID & Product & Type & Manufacturer \\
\hline AP & AQUA PATCH & $\mathrm{H}_{2} \mathrm{O}$ Reactive & Aqua Patch Road Materials, LLC \\
\hline $\mathrm{AQ}$ & Aquaphalt 6.0 & $\mathrm{H}_{2} \mathrm{O}$ Reactive & Roadstone Production, LLC \\
\hline $\mathrm{CCO}$ & ChemCo Asphalt Repair & Cutback & ChemCo Systems \\
\hline EZ & EZ Street ${ }^{\circledR}$ & Cutback & The EZ Street Company \\
\hline IA & Instant Asphalt' ${ }^{\mathrm{TM}} 6.0$ & $\mathrm{H}_{2} \mathrm{O}$ Reactive & Pavemend \\
\hline IRR & Instant Road Repair ${ }^{\circledR}$ & Cutback & International Roadway Research \\
\hline $\mathrm{PP}$ & Perma-Patch ${ }^{\circledR}$ & Cutback & Perma-Patch, Inc. \\
\hline QR & QUIKRETE ${ }^{\circledR}$ High Performance Blacktop Repair & Cutback & QUIKRETE ${ }^{\circledR}$ \\
\hline $3 R$ & Ready Road Repair ${ }^{\circledR}$ & Cutback & Gardner-Gibson ${ }^{\circledR}$ \\
\hline
\end{tabular}

Table 2.2 and Figure 2.1 provide gradations measured on solvent-extracted aggregate for all cold mixes except AP and EZ. The AP gradation was not attainable because aggregate could not be extracted from the mixture in traditional manners (e.g., ignition oven, solvent extraction) due to the water-reactive binder. Gradations are provided for AQ and IA, also waterreactive formulations, because a gradation band was provided in the manufacturers' technical data sheets; the median gradation for each band was identical for AQ and IA and is reported in Table 2.2 and Figure 2.1. The EZ gradation was not performed due to laboratory logistical issues. 
Table 2.2. Cold mix asphalt gradations by material.

\begin{tabular}{|c|c|c|c|c|c|c|c|c|}
\hline \multicolumn{2}{|c|}{ Property } & $\mathrm{AQ}$ & $\mathrm{CCO}$ & IA & IRR & PP & QR & $3 R$ \\
\hline \multicolumn{2}{|c|}{ NMAS } & 4.75 & 9.5 & 4.75 & 9.5 & 9.5 & 12.5 & 9.5 \\
\hline \multirow{13}{*}{ 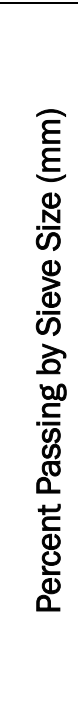 } & 50.0 & 100 & 100 & 100 & 100 & 100 & 100 & 100 \\
\hline & 37.5 & 100 & 100 & 100 & 100 & 100 & 100 & 100 \\
\hline & 25.0 & 100 & 100 & 100 & 100 & 100 & 100 & 100 \\
\hline & 19.0 & 100 & 100 & 100 & 100 & 100 & 100 & 100 \\
\hline & 12.5 & 100 & 100 & 100 & 100 & 100 & 100 & 100 \\
\hline & 9.5 & 100.0 & 100 & 100.0 & 99.7 & 100 & 88.3 & 100 \\
\hline & 4.75 & 92.5 & 83.1 & 92.5 & 81.6 & 86.8 & 37.0 & 69.6 \\
\hline & 2.36 & 37.5 & 18.1 & 37.5 & 46.7 & 26.0 & 10.4 & 15.2 \\
\hline & 1.18 & 22.0 & 5.3 & 22.0 & 31.1 & 7.6 & 5.4 & 5.7 \\
\hline & 0.60 & -- & 3.7 & --- & 23.2 & 4.9 & 4.3 & 4.0 \\
\hline & 0.30 & -- & 3.3 & -- & 16.9 & 3.9 & 3.6 & 3.4 \\
\hline & 0.15 & --- & 3.1 & --- & 11.6 & 3.4 & 3.1 & 3.1 \\
\hline & 0.075 & 4.5 & 2.8 & 4.5 & 7.7 & 2.9 & 2.7 & 2.6 \\
\hline
\end{tabular}

-- Gradations for AQ and IA were obtained from manufacturers' technical data sheets since as-received gradations could not be attained. Percent passing values were not provided between 1.18 and 0.075 $\mathrm{mm}$.

Figure 2.1. Cold mix asphalt gradations.

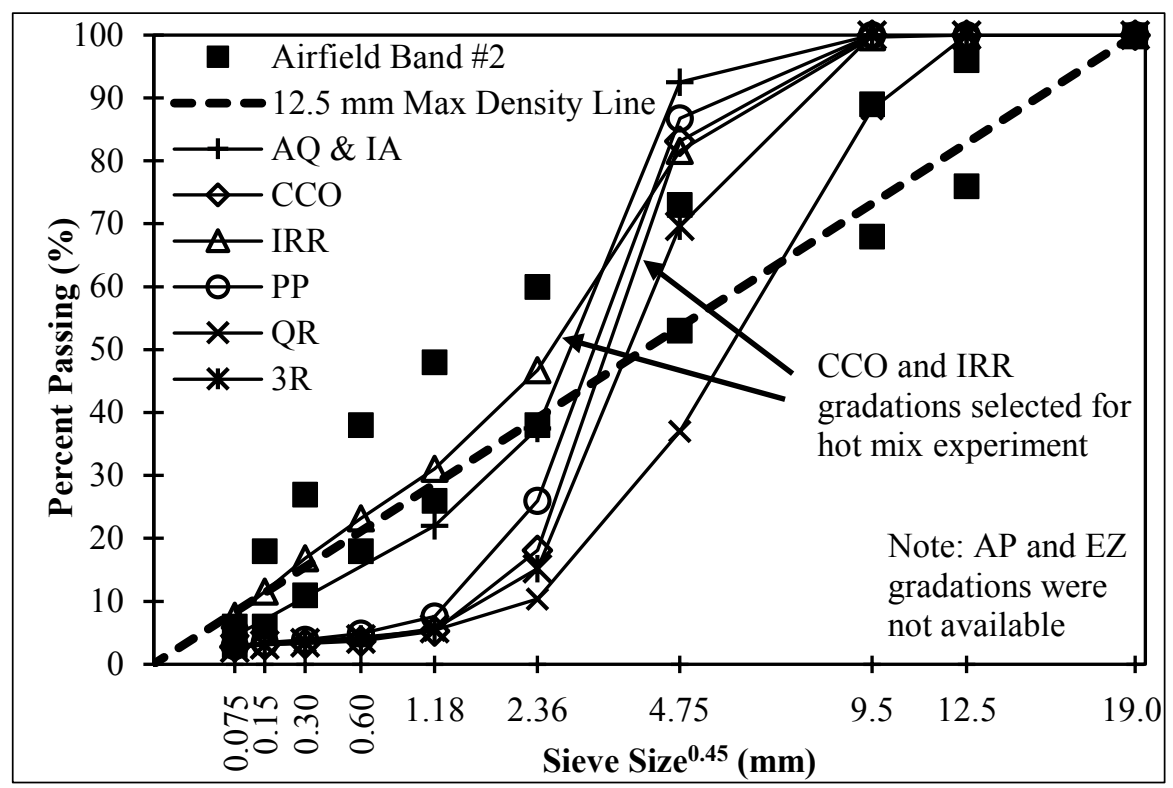

The maximum density line for a $12.5 \mathrm{~mm}$ nominal maximum aggregate size (NMAS) gradation is shown in Figure 2.1 since $12.5 \mathrm{~mm}$ is the largest NMAS tested. Individual plots for 4.75, 9.5, and 12.5 mm NMAS gradations were omitted for brevity as Figure 2.1 provides a depiction of each gradation for comparison. Most cold mixes are gap-graded and predominantly fall on the 
coarse side of their corresponding maximum density line. IRR, plotting mostly along the $9.5 \mathrm{~mm}$ NMAS max density line, was the key exception to this trend. Two 9.5 mm NMAS gradations, CCO and IRR, were selected for later evaluation in a hot mix asphalt experiment. IRR was selected because it was a finer gradation, while $\mathrm{CCO}$ was selected because it was representative of the gapped gradations.

\subsubsection{Hot mix asphalt materials}

Two HMA materials were used in this study as control mixtures for cold mixes. One material was produced and tested in the laboratory, while the other was produced and tested in the field. These two materials were designated as either CM-L or CM-F for laboratory or field control mixture, respectively.

CM-L consisted of raw materials (i.e., aggregates and asphalt binder) sampled during paving work at the Federal Aviation Administration (FAA) National Airport Pavement Test Facility (NAPTF) in Atlantic City, New Jersey. These raw materials were used to create three laboratory-produced, laboratory-compacted mixtures at various gradations denoted $\mathrm{NJ}, \mathrm{CCO}$, and IRR. Each CM-L mixture was identified by its gradation (e.g., CM-L-NJ, CM-L-CCO). CM-L-NJ was the original mixture used at the FAA facility in New Jersey and was a 75-blow Marshall-designed mixture. CM-L-NJ and CM-L-IRR were produced with $5.0 \%$ asphalt content $(P b)$, while CM-L-CCO was produced with $3.0 \% P_{b}$. The design $P_{b}$ was used for CM-L-NJ, while the asphalt contents for the CCO and IRR gradations were selected based on engineering judgment. Table 2.3 provides design properties for CM-L-NJ, while Figure 2.2 provides its gradation. CM-L materials were used as a control for cold mixes during the laboratory component of this study.

Table 2.3. CM-L-NJ design properties.

\begin{tabular}{|l|l|}
\hline Property & Value \\
\hline Mix Temp $\left({ }^{\circ} \mathrm{F}\right)$ & 325 \\
\hline Compaction Temp $\left({ }^{\circ} \mathrm{F}\right)$ & 305 \\
\hline Binder & PG 76-22 \\
\hline$P_{b}(\%)$ & 5.0 \\
\hline$G_{m m}\left(\mathrm{~g} / \mathrm{cm}^{3}\right)$ & 2.622 \\
\hline$V_{a}(\%)$ & 3.4 \\
\hline VMA $(\%)$ & 15.7 \\
\hline Stability $(\mathrm{lbs})$ & 3,950 \\
\hline Flow $(0.1$ in) & 12 \\
\hline
\end{tabular}


Figure 2.2. CM-L-NJ gradation.

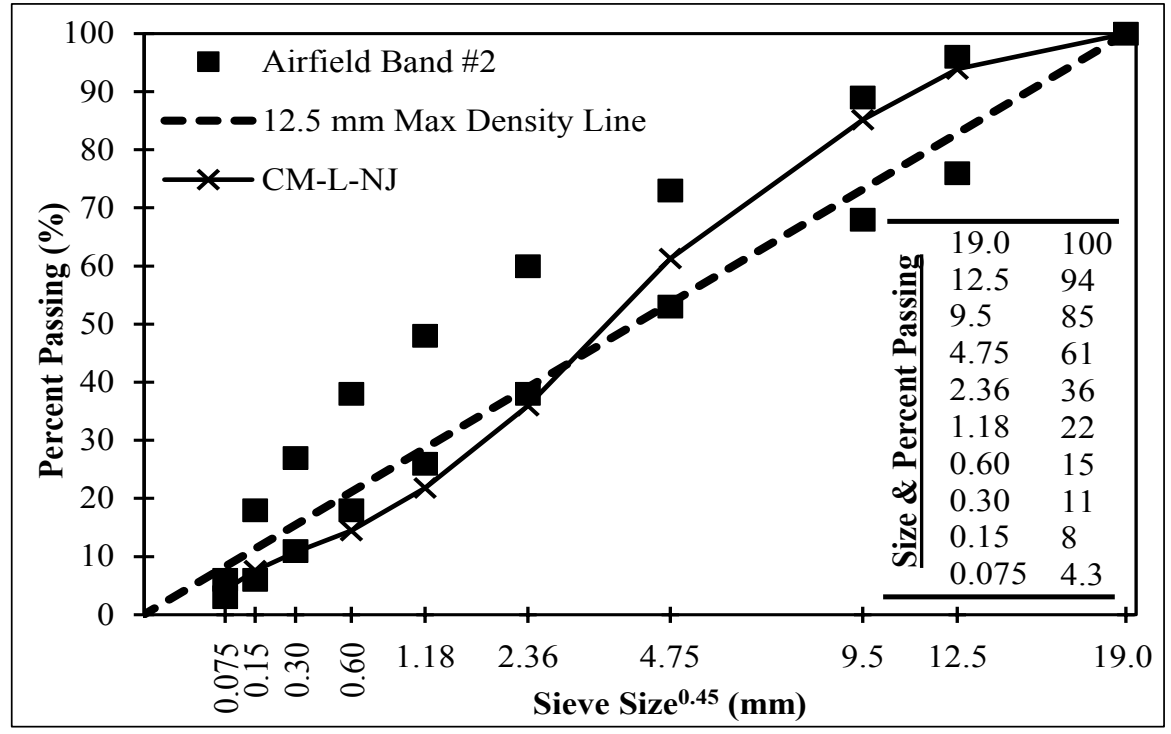

CM-F was a plant-produced, field-compacted warm mix asphalt (WMA) with $25 \%$ reclaimed asphalt pavement (RAP) provided by the APAC Mississippi plant in Vicksburg. It was used in this study as a control for cold mixes during the field component of this study and was treated as a hot mix, rather than warm mix, asphalt during this study, meaning it was heated to approximately $300{ }^{\circ} \mathrm{F}$ during testing. Detailed laboratory characterization of the CM-F sample was not conducted; limited properties were available from the supplier. Design $P_{b}$ was 6.0\% using a PG67-22 binder; specific gravities and volumetrics were not provided. Figure 2.3 provides the design CM-F gradation which was a 9.5 mm NMAS.

Figure 2.3. CM-F gradation.

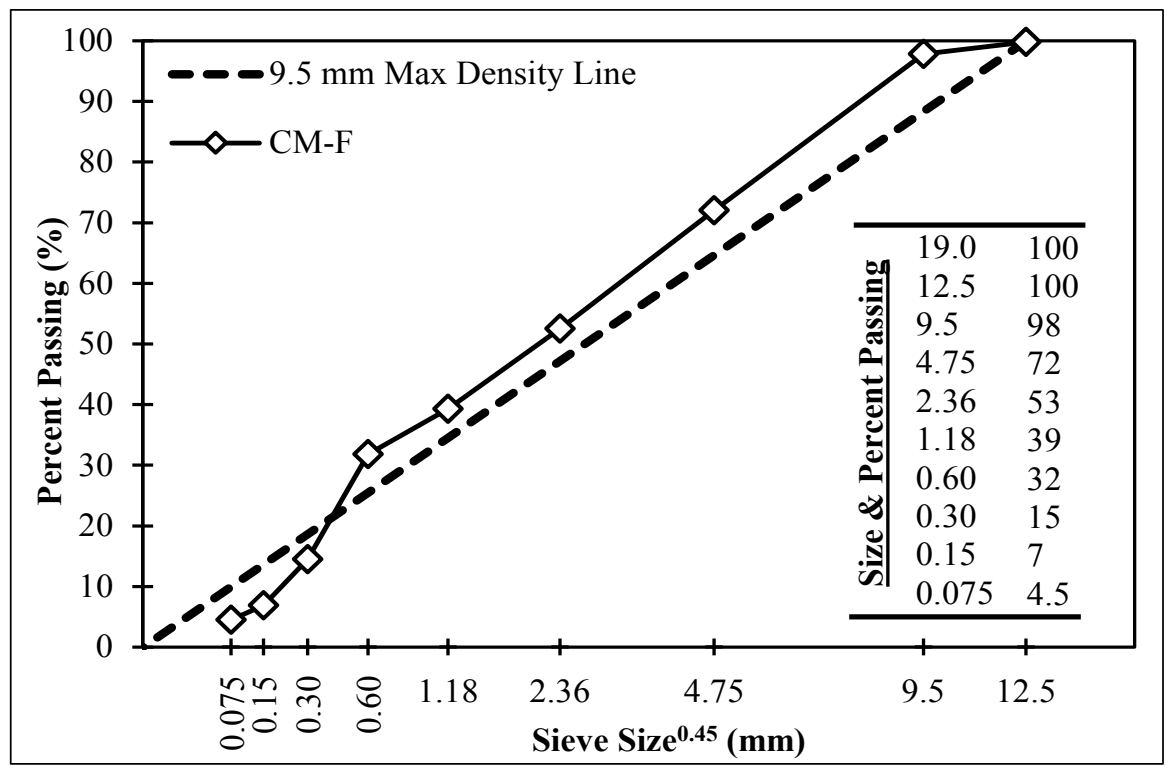




\subsection{Laboratory test methods}

\subsubsection{Mixing and compaction}

Cold mixes were stored in sealed buckets or bags indoors until used and were mixed, if necessary, according to manufacturer directions (e.g., water-activated mixtures). Preliminary testing was conducted to adjust target mix masses in order to produce $150 \mathrm{~mm}$ diameter specimens with target heights of either $75 \mathrm{~mm}$ or $115 \mathrm{~mm}$. Compaction was performed with 50 blows per side of an automatic Marshall hammer. Specimens were compacted at ambient temperature, immediately extruded from molds, and placed on mold base plates on a lab bench to cure.

CM-L mixes were batched from four bulk aggregate sources to the NJ gradation. In order to create batches for the CCO or IRR gradations, material batched to the NJ gradation was sieved into nine size fractions from $12.5 \mathrm{~mm}$ to finer than $0.075 \mathrm{~mm}$ and then batched to the CCO or IRR gradation. Aggregate was heated to $310^{\circ} \mathrm{F}$, mixed with PG 64-22 asphalt binder to the corresponding mixture's $P_{b}$, then compacted with 50 blows per side in the automatic Marshall hammer. Note that compaction effort, at 50 blows per side, was consistent for all mixtures even though CM-L-NJ was designed using a manual hammer at 75 blows per side. Similarly, PG 64-22 binder was substituted for the PG 76-22 binder used in CM-L-NJ design. Specimens were immediately extruded from molds and placed on base plates on a lab bench under a fan to cool. Figure 2.4 illustrates mixing and compaction equipment as well as the items typical of the mixing and compaction procedure.

\subsubsection{Curing}

Cold mix specimens were cured on a lab bench at ambient conditions for various cure times. Most commonly, specimens were cured overnight and then tested. Specific timing for curing of overnight specimens was not maintained, and actual cure times varied from approximately 18 to $28 \mathrm{hr}$. For purposes of this report, specimens cured overnight are nominally referred to as 1-day specimens. Additionally, some specimens were also cured for 3 and 5 days. 
Figure 2.4. Photographs of laboratory mixing and compaction: (a) automatic Marshall compactor, (b) HMA bucket mixer, (c) Marshall mold filled with mix, (d) specimens on lab bench.

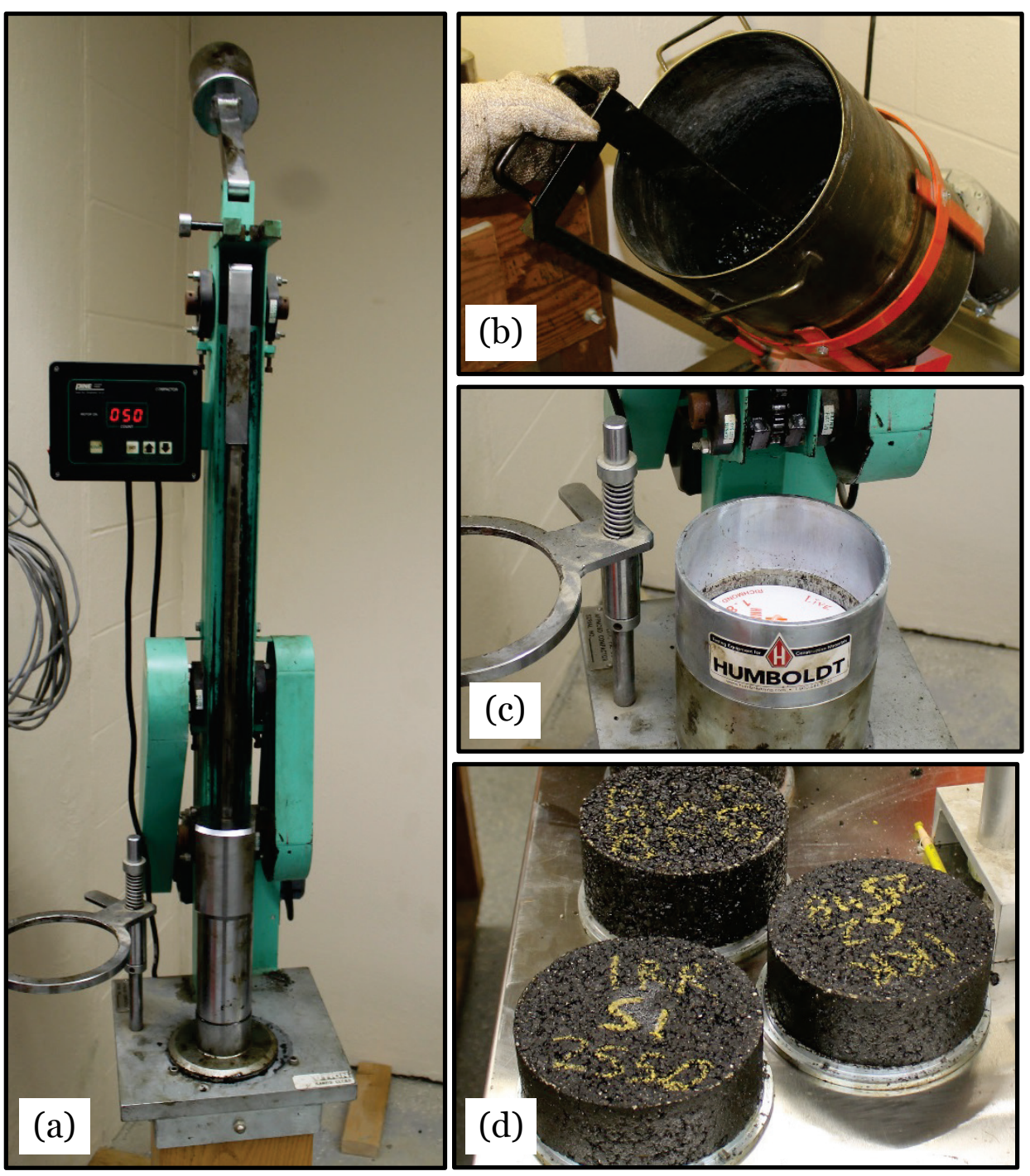

\subsubsection{Density measurement}

Theoretical maximum specific gravity $\left(G_{m m}\right)$ according to ASTM D2041 and bulk specific gravity $\left(G_{m b}\right)$ according to AASHTO T331 were measured on loose mixture and compacted specimens, respectively. $G_{m m}$ sample preparation varied depending on whether cold mix or HMA was being tested. HMA $G_{m m}$ samples were prepared based on typical practices (i.e., mix binder and aggregates, spread into loose mix while cooling). HMA $G_{m m}$ samples were not short-term aged before testing. All HMA mixtures were measured for $G_{m m}$. Cold mix $G_{m m}$ samples were batched from their buckets or bags and spread out on trays then placed in an oven at $140^{\circ} \mathrm{F}$ for 4 days to promote solvent removal and, consequently, $G_{m m}$ samples that were at 
constant mass. As with the gradations discussed in Section 2.1.1, $G_{m m}$ was not measured on water-reactive cold mixes or on EZ due to laboratory logistical issues.

$G_{m b}$, using the CoreLok ${ }^{\circledR}$ device, was measured on specimens for which $G_{m m}$ was also measured. Cold mix specimens, in particular, were measured for $G_{m b}$ after either one day of curing or on the final day of their curing protocol (e.g., at 5 days). Difficulties in vacuum sealing were sometimes encountered with cold mix specimens because the mixtures were relatively soft and easily deformed by the CoreLok bag as it sealed around a specimen (Figure 2.5). Care was taken to alleviate this problem to the extent possible by aligning the bag and specimen such that folds in the bag were less likely to occur.

Figure 2.5. Typical cold mix specimen deformed by CoreLok bag.

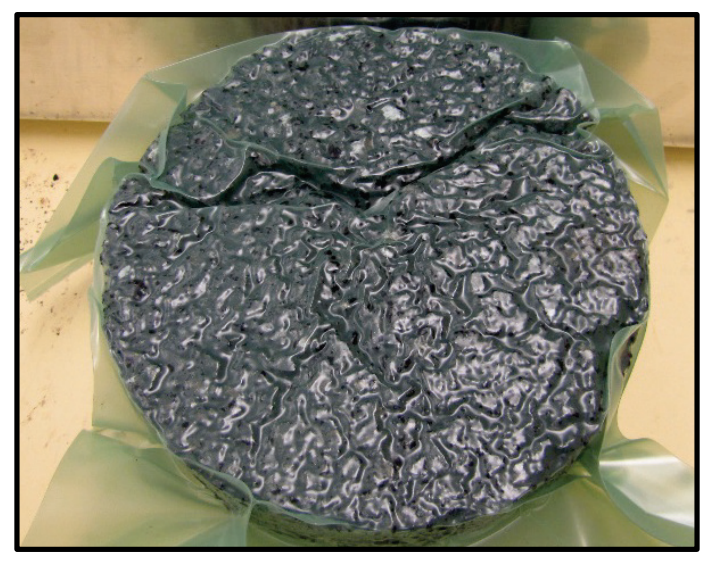

Air voids $\left(V_{a}\right)$ were calculated from $G_{m m}$ and $G_{m b}$ for a general understanding of mixture compactibility under a fixed compaction effort. Given the concerns presented, especially for CoreLok $G_{m b}$ of cold mix specimens, $V a$ values should be interpreted loosely. However, while not expected to be nearly as precise as for HMA, cold mix $V_{a}$ values can still be useful for general comparisons.

\subsubsection{Laboratory rut testing}

Mixtures evaluated in this study were tested for rutting behaviors in the Asphalt Pavement Analyzer (APA) shown in Figure 2.6. Tests were run in general accordance with AASHTO T340 to 8,000 cycles (16,000 passes). Test parameters were as follows: $100 \mathrm{lb}$ vertical load, 100 psi hose pressure, and $43{ }^{\circ} \mathrm{C}\left(110^{\circ} \mathrm{F}\right)$ test temperature. $\mathrm{T} 340$ requires specimens to be 
conditioned at the test temperature for 6 to $24 \mathrm{hr}$; in this study, specimens were generally placed in an oven or in the APA overnight at the test temperature and tested the following morning.

Figure 2.6. Asphalt pavement analyzer: (a) overall, (b) inside of cabin.

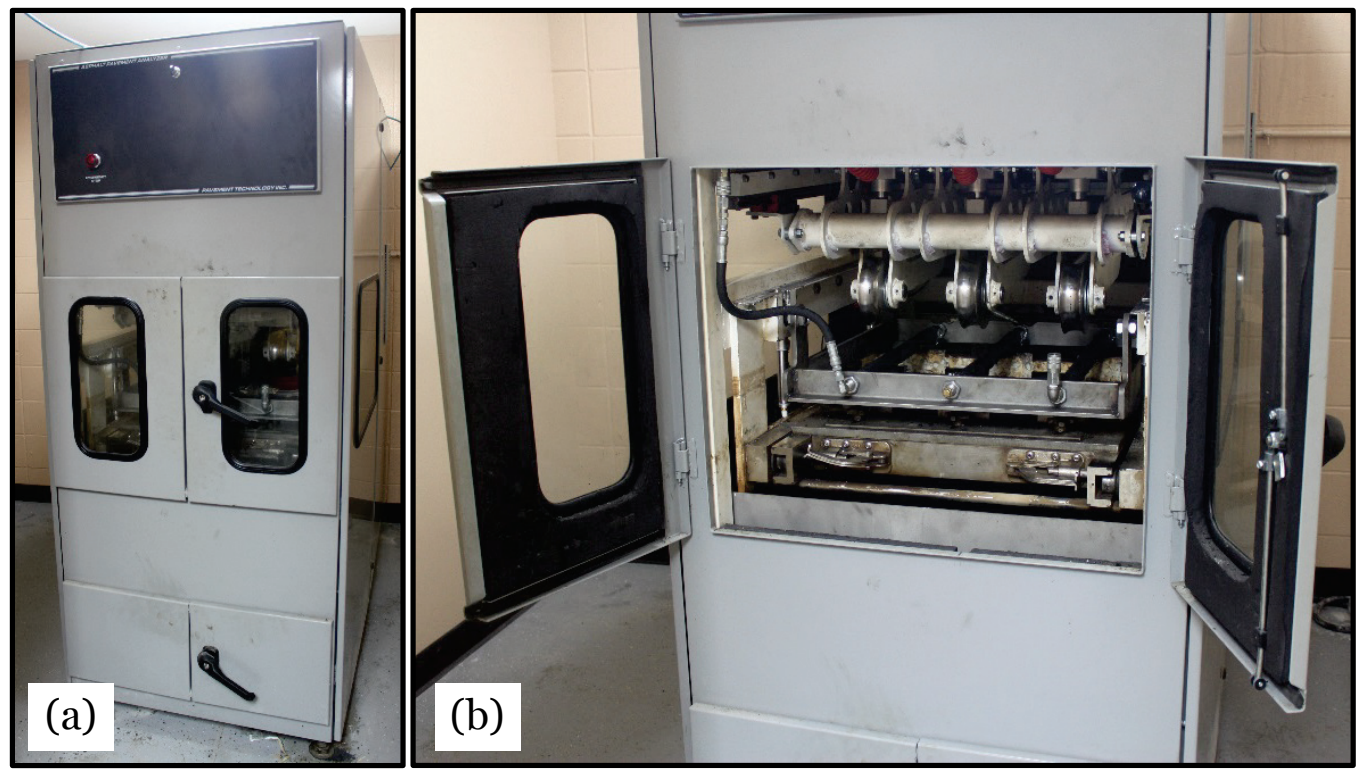

Note that APA molds were modified so that each mold's inside diameter was slightly enlarged. This could meaningfully affect results relative to typical $\mathrm{T}$ 340 procedures because specimens were not confined (i.e., mold diameters were larger than specimen diameters even after mold halves were completely tightened). Consequently, the modified molds permitted specimens to deform outward under load, meaning rutting could occur via two mechanisms - mixture shear failure (typical rutting mechanism) and outward deformation. This should be considered when evaluating results relative to other APA data outside of this report. Additionally, data collection points were improperly configured relative to default APA spacing. While this would have minimal impact on data comparisons within this report, care must be taken when comparing with APA data outside this report, as results may vary slightly. Improper configuration of the data collection points was not observed until after all testing for this report was complete.

Figure 2.7 provides a typical photograph of a tested APA specimen in an APA mold. A type of screening process was used when determining the APA test plan. All cold mixes were tested after one day of curing. For 3-day and 5-day APA testing, only select mixes were tested based on both 1-day APA results and a more subjective assessment after handling the mixtures in the laboratory, as discussed in the laboratory results chapter. 
Figure 2.7. Typical tested APA specimen.

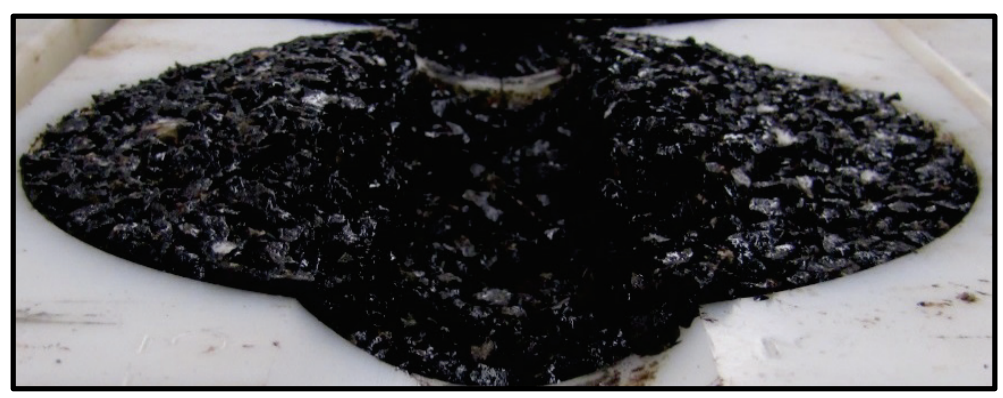

Table 2.4 summarizes the APA test plan for cold mixes. After 1-day testing, $\mathrm{CCO}$ and $\mathrm{QR}$ were removed from consideration, while all other mixtures were tested further to determine if additional curing could meaningfully impact rutting characteristics. After 3-day testing, EZ, PP, and 3R were removed from consideration, while AP, AQ, IA, and IRR were again further tested after five days of curing. Six replicate specimens were tested for each mixture at each cure time yielding a total of 120 cold mix specimens (20 APA tests) tested in this study.

Table 2.4. APA test plan for cold mixes.

\begin{tabular}{|l|c|c|c|}
\hline ID & 1-day Cure & 3-day Cure & 5-day Cure \\
\hline AP & $\checkmark$ & $\checkmark$ & $\checkmark$ \\
\hline AQ & $\checkmark$ & $\checkmark$ & $\checkmark$ \\
\hline CCO & $\checkmark$ & -- & -- \\
\hline EZ & $\checkmark$ & $\checkmark$ & -- \\
\hline IA & $\checkmark$ & $\checkmark$ & $\checkmark$ \\
\hline IRR & $\checkmark$ & $\checkmark$ & $\checkmark$ \\
\hline PP & $\checkmark$ & $\checkmark$ & -- \\
\hline QR & $\checkmark$ & -- & -- \\
\hline 3R & $\checkmark$ & $\checkmark$ & -- \\
\hline
\end{tabular}

For HMA mixtures, six replicates were also tested at the same test parameters as cold mix specimens. Curing time was not a variable for HMA mixtures; therefore, one set of six specimens was tested per mixture. Collectively for CM-L-NJ, CM-L-CCO, and CM-L-IRR, this yielded 18 total HMA specimens (3 APA tests) tested in this study.

\subsubsection{Indirect tensile strength testing}

Indirect tensile strength (IDT) testing was conducted in general accordance with ASTM D6931. IDT testing comprised a small amount of the overall 
project's testing effort as only one cold mix, AQ, was tested for IDT strength $\left(S_{t}\right)$. AQ specimens were cured for 1,3 , and 5 days; three replicates were tested at each cure time.

\subsection{Field test methods}

\subsubsection{Phase 1 field testing}

One HMA and three cold mix materials were tested during Phase 1 field testing beginning July 1, 2015: CM-F, AQ, IA, and IRR. CM-F was tested as the HMA control. AQ and IA were tested based on favorable laboratory APA results. Note that AP performed very favorably according to APA testing but was unable to be field tested in Phase 1 since the additional quantities needed for field testing were not provided in time by the material supplier. Lastly, IRR was included in Phase 1 field testing since it is a currently approved product. The mean daily temperature during Phase 1 field testing ranged from 80 to $84^{\circ} \mathrm{F}$, the minimum daily temperature ranged from 71 to $78^{\circ} \mathrm{F}$, and the maximum daily temperature was $89^{\circ} \mathrm{F}$.

Field testing was conducted within ERDC facilities in an existing asphalt test section that had been in place for several years. Areas for patching were created by saw cutting 2 -ft squares to a depth of 4 in. Patch locations were situated in a three-by-four grid pattern so that three curing protocols could be investigated for the four materials tested (12 patches total). Each set of four materials is further referred to as a series. The grid pattern was spaced such that trafficking of an entire series could occur simultaneously.

Patches were placed with hand tools in two approximately equal lifts of material. For the water-activated cold mixes, AQ and IA, water was added according to manufacturer recommendations. Cold mix compaction was achieved via a hand tamp and a Northern Industrial Equipment JPC-80 vibratory plate compactor (PC). HMA compaction was achieved via a CAT walk-behind steel wheel roller (CR). Figure 2.8a shows an example of a compacted field patch. In-place densities were not obtained.

Each series of four patches was subjected to one of three curing protocols prior to trafficking. These protocols are referred to as Conditions 1, 2, and 3 , and are denoted $\mathrm{C}_{1}, \mathrm{C}_{2}$, and $\mathrm{C}_{3}$ in this report. Condition 1 called for a 2-hr ambient-temperature cure time. Condition 2 utilized a 2-hr cure time that included 20 min of infrared heating per patch, not including the CM-F patch, and 100 min at ambient temperature. A skid steer-mounted 
Heatwurx HWX-30 heater (Figure 2.9) was outfitted with a thermostat to maintain a relatively constant temperature of $375^{\circ} \mathrm{F}$. Infrared heating increased patch surface temperatures to approximately $155^{\circ} \mathrm{F}$ after 20 min. Infrared heat was applied to the first patch beginning immediately after compaction so that the fourth patch had time to cool to approximately ambient pavement temperatures. Condition 3 utilized a 72$\mathrm{hr}$ ambient-temperature cure time.

Figure 2.8. Example field patch (IA shown): (a) before trafficking, (b) after trafficking.

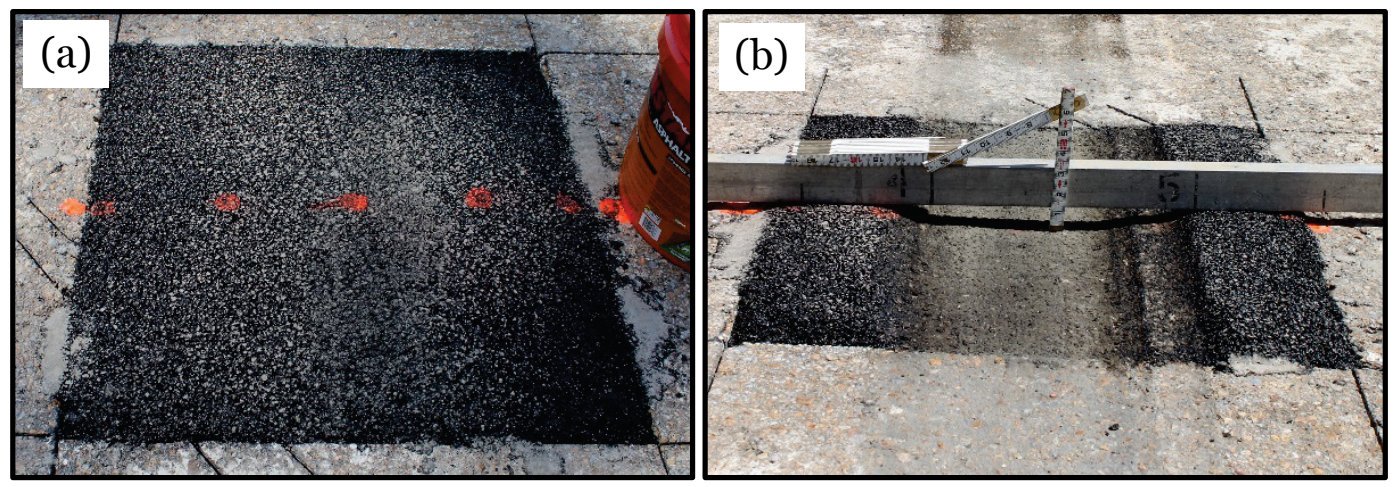

Figure 2.9. Heatwurx infrared heater.

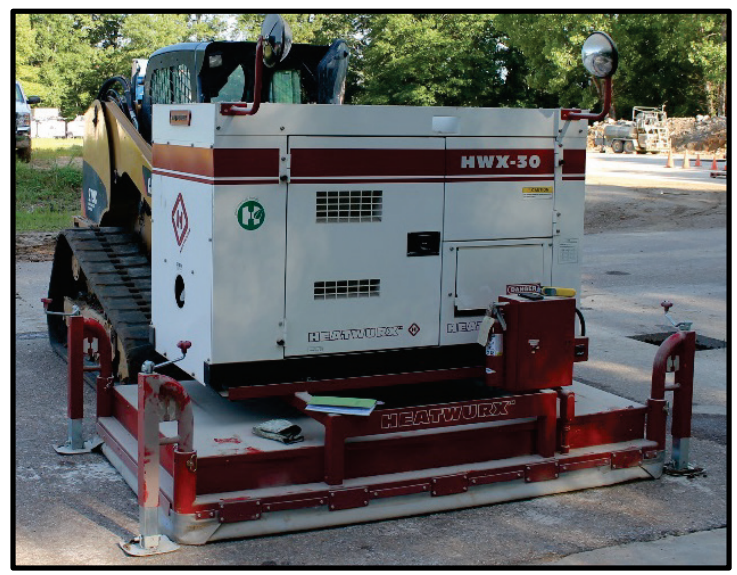

Trafficking was conducted with a load cart outfitted with an F-15E tire as shown in Figure 2.10. The load cart was designed to simulate the maximum single-wheel F-15E gross loading of 35,235 lb at a tire pressure of $325 \mathrm{psi}$. A channelized traffic pattern was used in this project. During trafficking, deterioration of the repair was monitored, and rutting measurements were recorded periodically based on the rate of deterioration. Rut measurements were taken as shown in Figure 2.8b by placing a straightedge across the rut and measuring the maximum rut depth. Trafficking was conducted to either failure, which was established as $1 \mathrm{in} .(25.4 \mathrm{~mm})$ of rutting, or 100 passes. 
Figure 2.10. F-15E load cart.

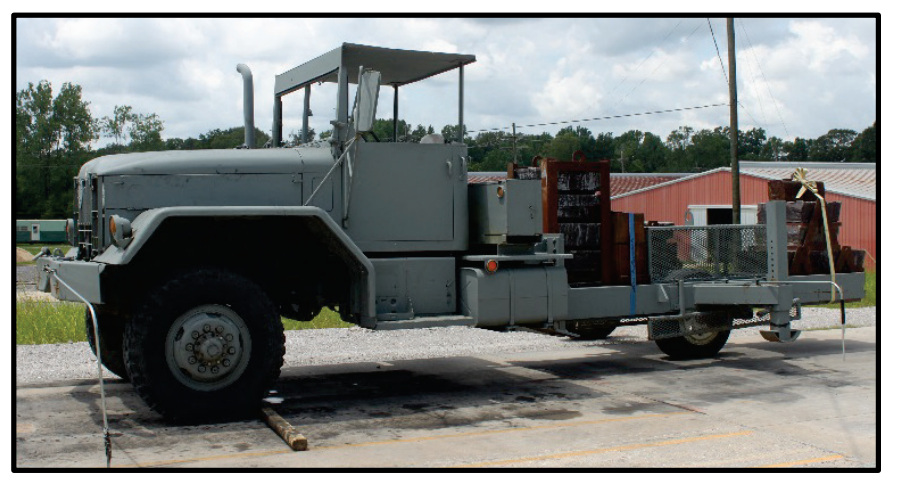

\subsubsection{Phase 2 field testing}

Three cold mix materials were tested during Phase 2 field testing beginning August 20, 2015: AP, AQ, and IA. Phase 2 field testing was conducted primarily to evaluate AP since it was not able to be included in Phase 1. AQ and IA were tested again for comparison of results; however, $\mathrm{CM}-\mathrm{F}$ and IRR were not tested a second time. The mean daily temperature during Phase 2 field testing ranged from 78 to $81^{\circ} \mathrm{F}$, the minimum daily temperature ranged from 73 to $78{ }^{\circ} \mathrm{F}$, and the maximum daily temperature ranged from 84 to $89^{\circ} \mathrm{F}$. Field testing procedures were generally similar between Phases 1 and 2 with only a few notable differences; for this reason, only procedures unique to Phase 2 are discussed in this section.

Two compaction methods were considered in Phase 2. Patches were compacted with either the PC as in Phase 1 or the CR, which was used in Phase 1 for CM-F only. A fourth curing condition (C4), $24 \mathrm{hr}$ ambient curing, was utilized. In all, six patches were tested (3 materials, 2 compaction methods, 1 curing condition).

Rut measurements were recorded periodically throughout trafficking with the F-15E load cart. Max rut depth measurements were obtained using the straightedge method described in Phase 1. Cross-section profiles were also obtained using a surveying rod and level. As in Phase 1, patches were trafficked until failure (25.4 $\mathrm{mm}$ of rutting) or 100 passes, whichever came first. 


\section{Laboratory Test Results}

This chapter presents results of laboratory-compacted specimens and is divided into three sections that provide density and air void results, APA rutting results, and IDT $S_{t}$ results.

\subsection{Density and air voids}

Table 3.1 presents average 50-blow density results for available cold mixes and all CM-L mixtures. Cold mix $V_{a}$ values range from approximately $17 \%$ to $32 \%$ depending on the material. Concerns exist regarding the validity of cold mix $G_{m m}$ and $G_{m b}$ values. In addition to normal testing variability, a measured $G_{m m}$ value of a cold mix is also dependent on the level of curing the sample has undergone, a variable that was not tightly controlled in this project. Residual solvent was routinely observed in the pycnometer during testing. Residual solvent in both $G_{m m}$ and $G_{m b}$ specimens is likely to affect the accuracy of the $V_{a}$ calculations. Similarly, the issues encountered with CoreLok measurements described in Section 2.2.3 could have meaningfully affected $G_{m b}$ measurements, though there is no means of quantifying these effects. For these reasons, $V_{a}$ results are not intended to be used to directly compare one cold mix to another. The purpose of showing cold mix $V_{a}$ results is primarily to demonstrate the difference in the range of cold mix $V_{a}$ values relative to conventional HMA with design-level $V_{a}$ 's around $4 \%$ and construction-target-level $V_{a}$ 's around 7 to $8 \%$. From this perspective, the difference between cold mix and typical HMA $V a$ values is significant.

Table 3.1. Laboratory density and air void results.

\begin{tabular}{|l|c|c|c|}
\hline ID & $G_{m m}\left(\mathrm{~g} / \mathrm{cm}^{3}\right)$ & $G_{m b}\left(\mathrm{~g} / \mathrm{cm}^{3}\right)$ & $V_{a}(\%)$ \\
\hline CCO & 2.676 & 1.879 & 29.8 \\
\hline IRR & 2.448 & 2.020 & 17.5 \\
\hline PP & 2.671 & 1.830 & 31.5 \\
\hline QR & 2.439 & 1.907 & 21.8 \\
\hline 3R & 2.607 & 1.769 & 32.1 \\
\hline CM-L-CCO & 2.691 & 2.017 & 25.0 \\
\hline CM-L-NJ & 2.620 & 2.383 & 9.0 \\
\hline CM-L-IRR & 2.573 & 2.401 & 6.7 \\
\hline
\end{tabular}

-- Recall that $G_{m m}$ was not obtained for AP, AQ, EZ, and IA. 
HMA Va values ranged from $6.7 \%$ to $25.0 \%$. Of the three CM-L mixtures, CM-L-NJ is the only designed mixture (others were laboratory-fabricated and did not follow any formal design procedure). Even still, CM-L-NJ was compacted with 50 blows per side in a 6-in. mold with a mechanical hammer instead of the 75 blows per side in a 4-in. mold with a manual hammer for which it was designed, which could partially explain its high 9.0\% Va value. Considering the mixture's design Va's average $3.4 \%$ (Table 2.3), it is not likely that decreasing compaction effort to 50 blows would fully account for the $5.6 \%$ Va increase. $\mathrm{Gmm}$, at 2.620 is reasonable compared to the Table 2.3 design Gmm of 2.622, which suggests the mixture itself is correct. Gmb is low relative to $\mathrm{Gmm}$, most likely suggesting either compaction issues (i.e., $\mathrm{Gmb}$ is in fact correct) or $\mathrm{Gmb}$ measurement issues (i.e., specimens had lower Va and Gmb is incorrect). Overall, CM-LNJ Va values appear questionable.

CM-L-IRR is the finest gradation tested of the CM-L mixtures. To be a laboratory-fabricated mixture in which its $P_{b}$ was not based on a design procedure, its $V_{a}$ of $6.7 \%$ appears reasonable. Given that CM-L-IRR utilized a finer gradation (more aggregate surface area), asphalt binder demand would be greater than it was for CM-L-NJ, all other factors being equal. While binder demand would be greater, CM-L-IRR was not provided any additional binder to satisfy this demand. Instead it was maintained at $5.0 \% P_{b}$, which would increase $V_{a}$ (relative to the $4 \%$ design $V_{a}$ target); therefore, CM-L-IRR $V_{a}$ 's appear reasonable.

CM-L-CCO is the coarsest gradation tested of the CM-L mixtures; thus, it would be expected to have the highest $V_{a}$. While it does, 25.0\% $V_{a}$ is unreasonably high. In this case, $G_{m m}$ appears that it could be too high, and $G_{m b}$ appears that it could be too low. As with the aforementioned explanations, a number of factors could have affected either measurement. Overall, CM-L-CCO $V_{a}$ values appear questionable. In either case, the creation of hot asphalt mixtures using replicated gradations from the cold mixes shows that aggregate gradation is a major factor influencing the compacted $V_{a}$ and the mix's ability to densify. Lack of adequate densification during compaction is a major factor in rutting behavior.

\subsection{APA rutting}

Figure 3.1 presents APA results for 1-day cured cold mix specimens. Cold mixes can be easily grouped into two general categories: 1 ) those that reached the maximum APA rut depth in less than 2,000 cycles, and 2) those 
that lasted 8,00o cycles. These two categories are further referred to as Group B and Group A products, respectively. Recall that APA testing in this report was conducted at $43{ }^{\circ} \mathrm{C}$ rather than the traditional $64{ }^{\circ} \mathrm{C}$ test temperature. Behaviors of these materials would likely worsen at $64^{\circ} \mathrm{C}$; this should be considered when discussing results (e.g., a $6 \mathrm{~mm}$ rut depth at 8,000 cycles and $43{ }^{\circ} \mathrm{C}$ would be greater with respect to standard $64{ }^{\circ} \mathrm{C}$ testing).

Figure 3.1. Cold mix 1-day APA results.

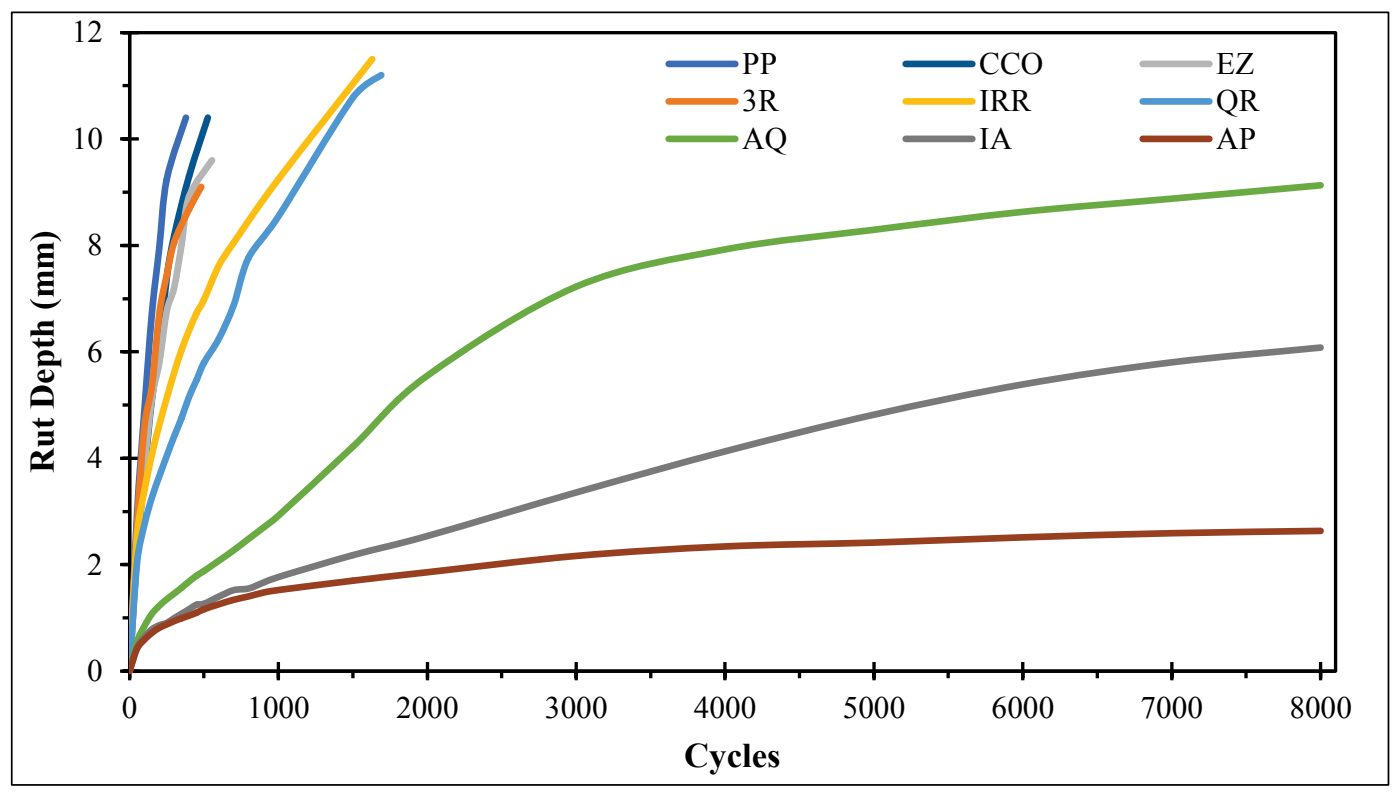

From a material selection perspective where rutting is the primary point of interest, there is little motivation to recommend a product failing at less than 2,000 cycles (Group B) for anything other than extreme emergency situations when there are products that exhibit significantly better rut characteristics (Group A). In normal operations, the group of products including AQ, IA, and AP would be preferred. It is of interest to note that IRR, the currently used cold mix material, is located in the less desirable group, suggesting that revisions to approved-product lists may be worth considering.

While early rutting performance is often of primary interest for airfield applications, understanding material behavior over time as the cold mixes cure is beneficial. Figure 3.2 presents 3-day results for comparison to 1-day results for select Group B cold mixes. Figure 3.3 presents 1-, 3-, and 5-day results for Group A cold mixes as well as IRR for comparison since IRR is the current recommended product. 
Figure 3.2. Cold mix 1 and 3 day APA results.

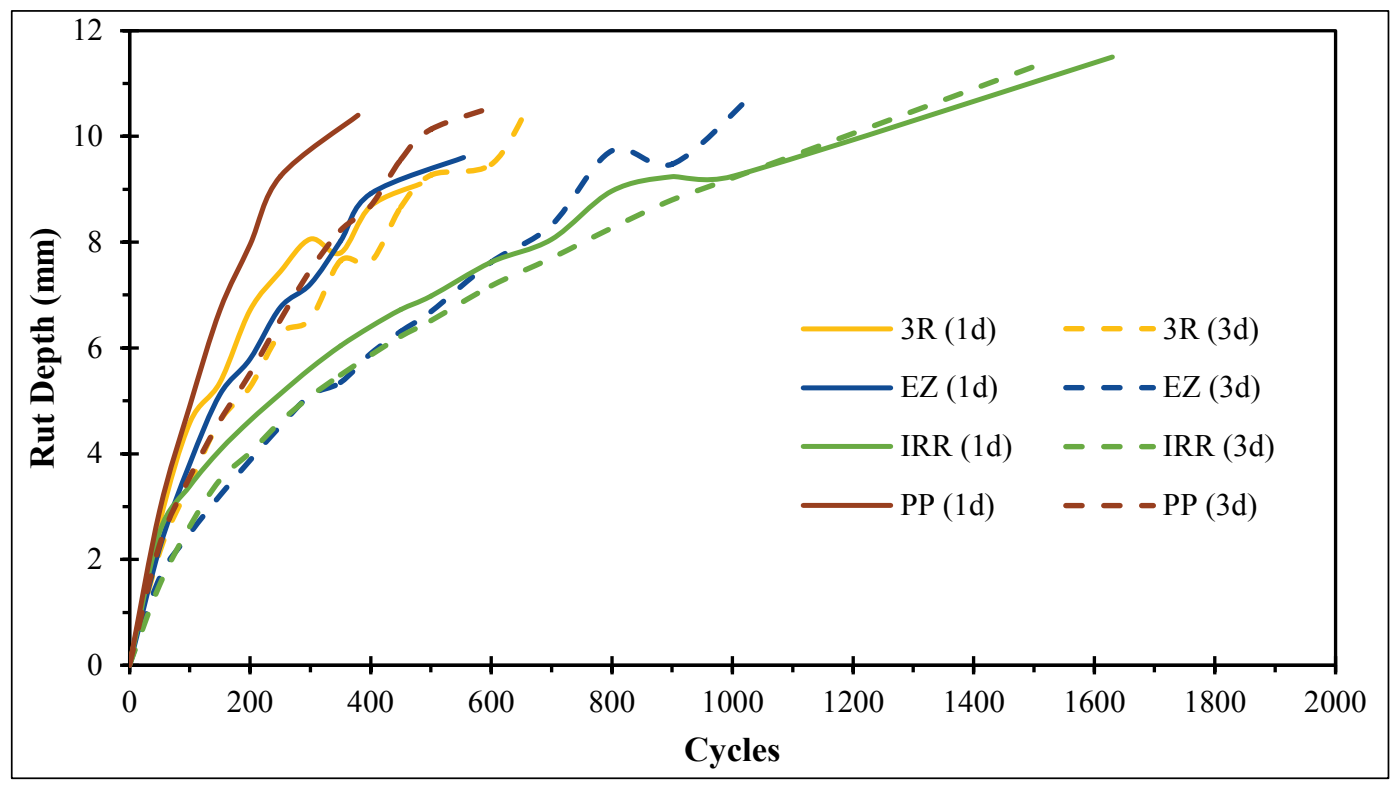

Figure 3.3. Cold mix 1, 3, and 5 day APA results.

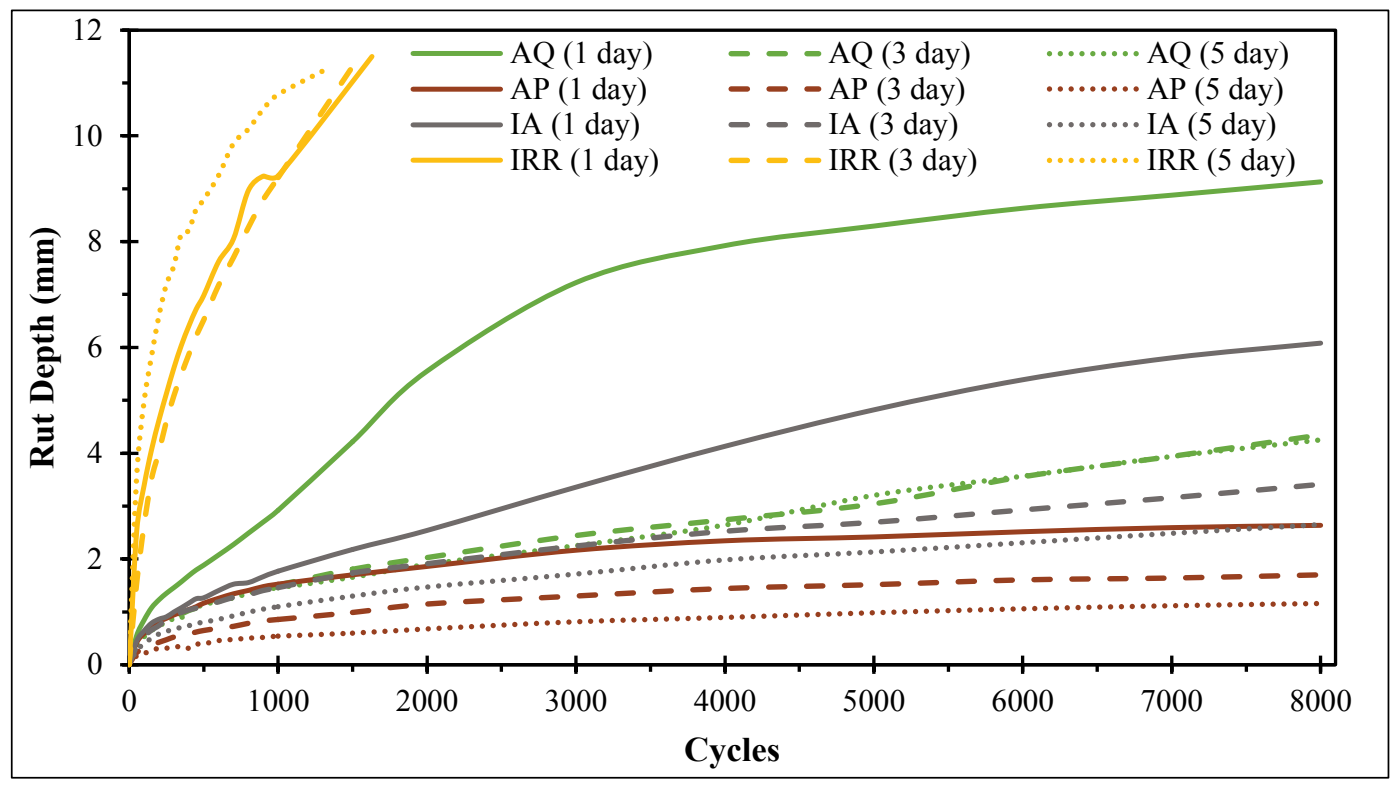

Figure 3.2 plots rut data to 2,000 cycles and illustrates that additional curing time did not meaningfully improve rutting characteristics for any of the Group B products. EZ's cycles to failure approximately doubled between 1 and 3 days of curing; however, increasing from 500 to 1,000 cycles is not meaningful considering the product, even at 3 days, only withstood oneeighth of a standard APA test at a reduced temperature. Other improvements with curing time were less noticeable than that of EZ or effectively nonexistent. 
Figure 3.3 demonstrates that additional curing time could have moderate effects on Group A products. IRR results were included for completeness. As with Figure 3.2, IRR 5-day results were not improved relative to 1- or 3 -day results and were likely within the variability of the material and test.

Group A rutting improvements were more noticeable between 1 and 3 days than between 3 and 5 days, which is intuitive. Improvements were fairly significant in some cases, as with AQ where rut depth decreased from approximately 9 to $4 \mathrm{~mm}$ from 1 to 3 days. For all Group A products, rut depths decreased 35 to $52 \%$ from 1 to 3 days and 2 to $32 \%$ from 3 to 5 days. Overall, Group A products ranked in order of AP, IA, and then AQ with respect to APA rutting performance.

Figure 3.4 provides APA results for the HMA control mixtures. The most informative result in terms of comparison with cold mixes is that of the fully-designed CM-L-NJ mixture. Its rut depth is approximately $4 \mathrm{~mm}$, and it assists in further assessing performance within Group A mixtures. From Figure 3.3 Group A mixtures, only AQ and IA 1-day results exceeded $4 \mathrm{~mm}$; all others exhibited rut depths of approximately $4 \mathrm{~mm}$ or less.

Figure 3.4. HMA control mixture APA results.

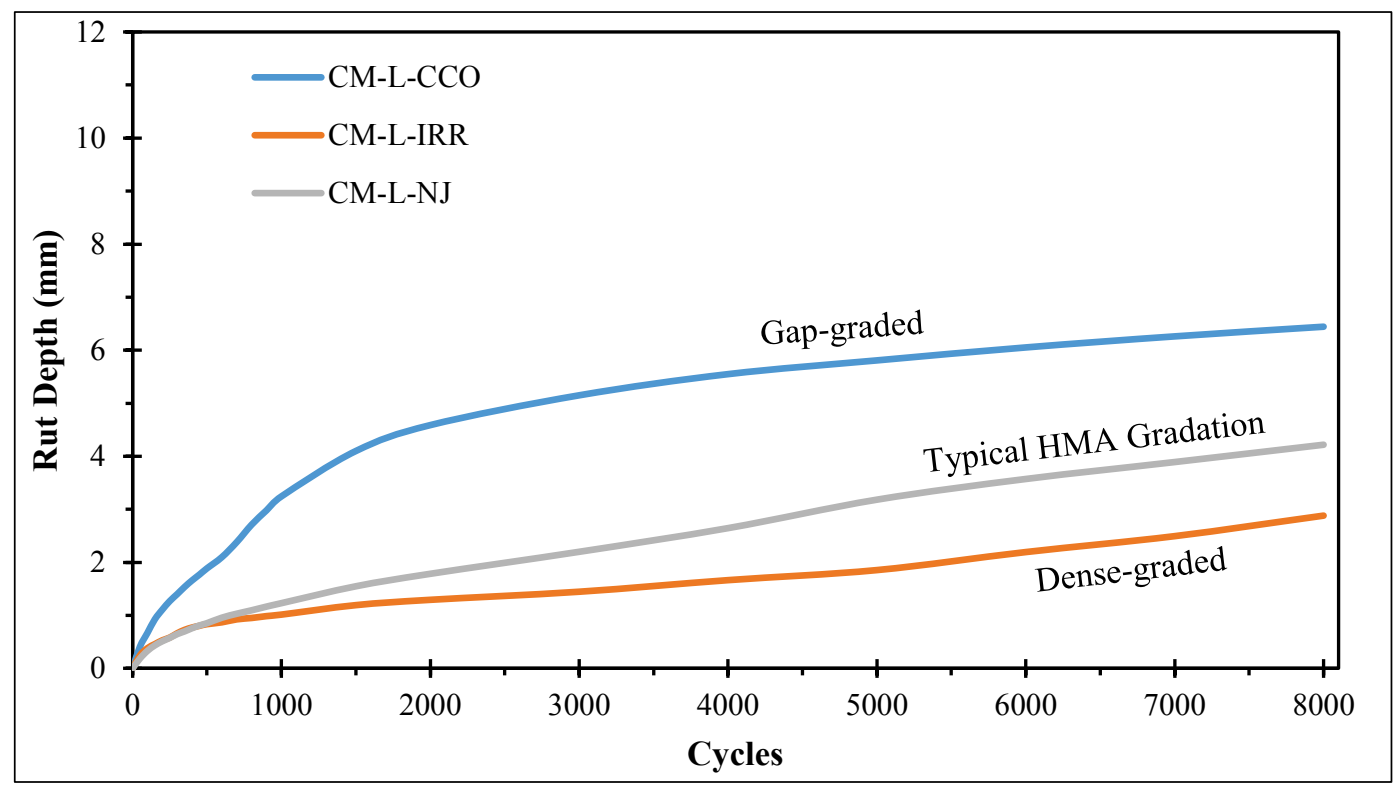

CM-L-CCO and CM-L-IRR results were presented to examine the effect of gradation on rutting performance with the intent of better understanding the performance of Group B products. Gradation can affect the overall stability of a mixture and its subsequent resistance to rutting. However, 
because of the potential issues with the laboratory-fabricated control mixtures, APA results of CM-L-CCO and CM-L-IRR should be considered questionable as actual $V_{a}$ values in addition to specimen integrity are not truly known for these mixtures.

\subsection{Indirect tensile strength}

Table 3.2 presents IDT $S_{t}$ data for AQ for a general understanding of cold mix $S_{t}$ relative to conventional asphalt. AQ, while not the most rutresistant material tested, was one of the Group A products, which is why it was selected. $S_{t}$ ranged from 8 to 12 psi and increased very slightly with cure time, though the trend is not strong.

Table 3.2. AQ IDT $S_{t}$ Results.

\begin{tabular}{|c|c|}
\hline Cure Time (days) & Avg $\boldsymbol{S}_{t}(\mathbf{p s i})$ \\
\hline 1 & 8 \\
\hline 3 & 12 \\
\hline 5 & 10 \\
\hline
\end{tabular}

For comparison, Rushing et al. (2013) presents $S_{t}$ data for multiple WMA mixes evaluated for use on heavy traffic airfields. For laboratory-produced WMA, $S_{t}$ values from 275 to 390 psi were reported. Similarly, for plantproduced WMA, $S_{t}$ values from 300 to 350 psi were reported. This illustrates the drastic difference in tensile strength behaviors between cold mixes tested in this project and conventional asphalt (WMA or HMA). 


\section{Field Test Results}

\subsection{Phase 1 field testing}

Figure 4.1 presents rutting results measured using a straightedge during Phase 1 field testing. Cold mix patches were generally arranged within the test grid pattern in order from best to worst rutting expectations based on laboratory results. This was done so that if the worst cold mix failed (reached $25.4 \mathrm{~mm}$ rut depth), trafficking of the remaining patches could continue unimpeded. If all cold mixes failed prior to reaching 100 passes, trafficking of CM-F was also stopped.

Figure 4.1. Phase 1 field rutting results by pass level.

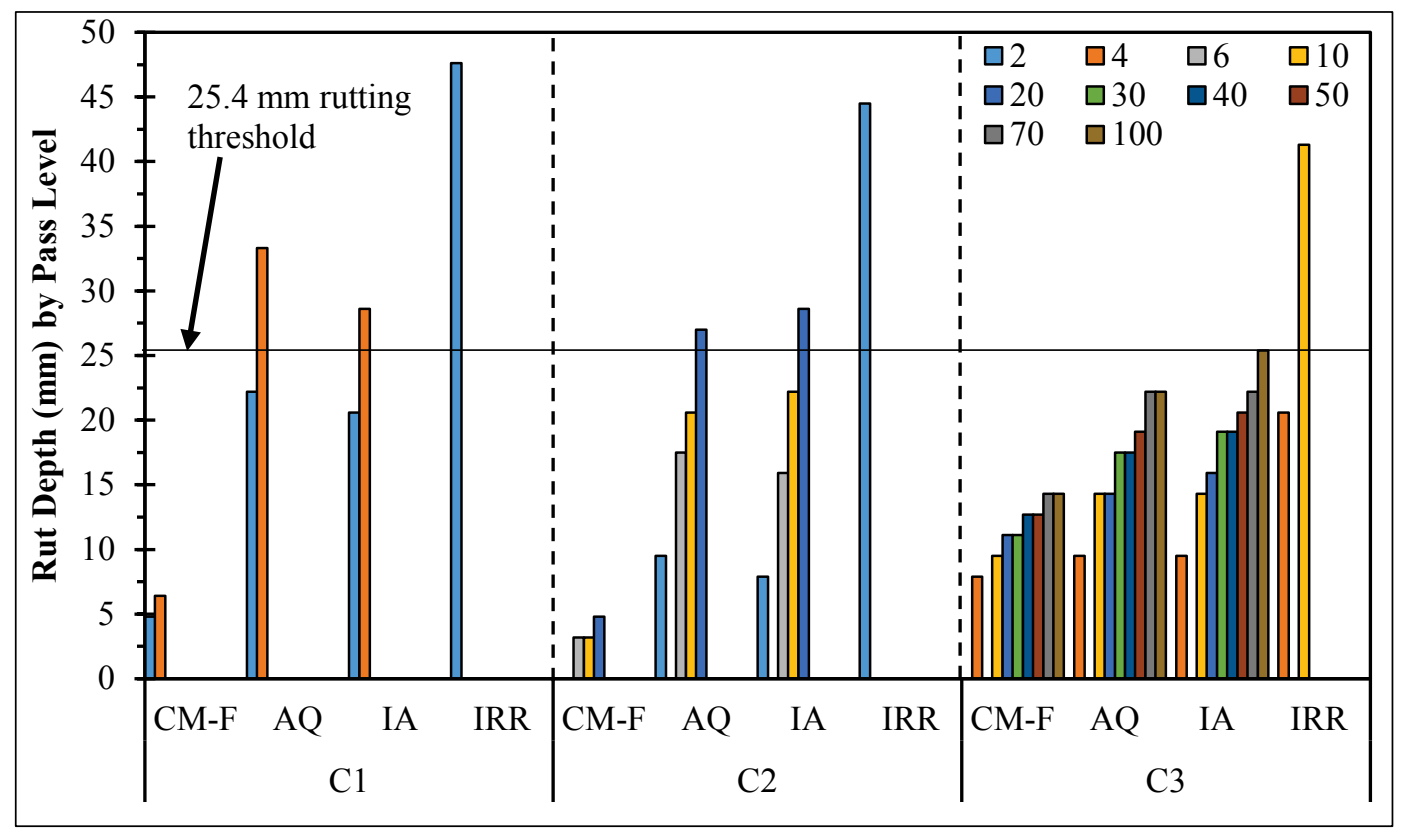

For $\mathrm{C} 1$ ( $2 \mathrm{hr}$ ambient curing), the currently recommended product IRR failed within just two passes of the load cart. Note that the rut depth after two passes was nearly double the failure threshold. AQ and IA performed similarly, but both failed after only four passes. Their performance was drastically different than that of CM-F, which was placed as a hot mixture.

For $\mathrm{C} 2$ ( $1 \mathrm{hr} 40 \mathrm{~min}$ ambient curing with 20 min infrared heating), IRR again failed within two passes; its rut depth improved very slightly, but not meaningfully, relative to C1. AQ and IA again performed fairly similarly, failing within 20 passes. Performance increased by a factor of five relative 
to $\mathrm{C} 1$; however, the number of passes to failure was still relatively low. By contrast, CM-F rut depths were less than $5 \mathrm{~mm}$ and were also lower than in $\mathrm{C}$. Recall that CM-F was not conditioned with infrared heating in $\mathrm{C} 2$ like cold mixes; therefore, $\mathrm{CM}-\mathrm{F}$ conditions were identical between $\mathrm{C} 1$ and C2. The slight differences in rut depth between $\mathrm{C} 1$ and $\mathrm{C} 2$ could be a result of in-place density differences from one patch to another or an increased pavement temperature at the time of $\mathrm{C}_{2}$ testing.

For $\mathrm{C}_{3}$ (72 hr ambient curing), IRR failed within 10 passes, which, while slightly better than performance in $\mathrm{C}_{1}$ or $\mathrm{C} 2$, was not a practically meaningful increase. AQ and IA performed closely and withstood 100 passes, meeting the objective pass level. Rut depths were greater with CM$\mathrm{F}$ relative to $\mathrm{C} 1$ and $\mathrm{C} 2$, perhaps partly due to lower densities or an increased pavement temperature at testing relative to $\mathrm{C} 1$, and would not have necessarily benefited from the additional curing time since HMA, unlike cold mixes, would be almost unaffected by ambient conditioning times in the 2 to $72 \mathrm{hr}$ range.

Overall, the beneficial effect of additional curing time on cold mixes, especially AQ and IA, is evident from Figure 4.1. AQ and IA outperformed IRR, particularly after a longer curing time. AQ and IA demonstrate a better curing efficiency in that the increase in rutting resistance with curing time is significantly greater than for IRR. While AQ rutted slightly less than IA after $\mathrm{C}_{3}$ conditioning and 100 passes, this difference is of little significance without additional replication.

\subsection{Phase 2 field testing}

Figure 4.2 presents rutting results measured using a straightedge during Phase 2 field testing. Phase 2 differed from Phase 1 in that CM-F was not tested again but AP was tested. Additionally, only one curing condition (C4) while two compaction methods (PC and CR) were considered.

As in Phase 1 field testing, AQ and IA performed similarly. Both failed within 30 passes for both PC and CR compaction. However, AP did not exceed the $25.4 \mathrm{~mm}$ rutting threshold after 100 passes. AP rutting behaviors were also similar for both $\mathrm{PC}$ and CR compaction. Overall, AP performed considerably better than $\mathrm{AQ}$ and IA with respect to rutting resistance. 
Figure 4.2. Phase 2 field rutting results by pass level.

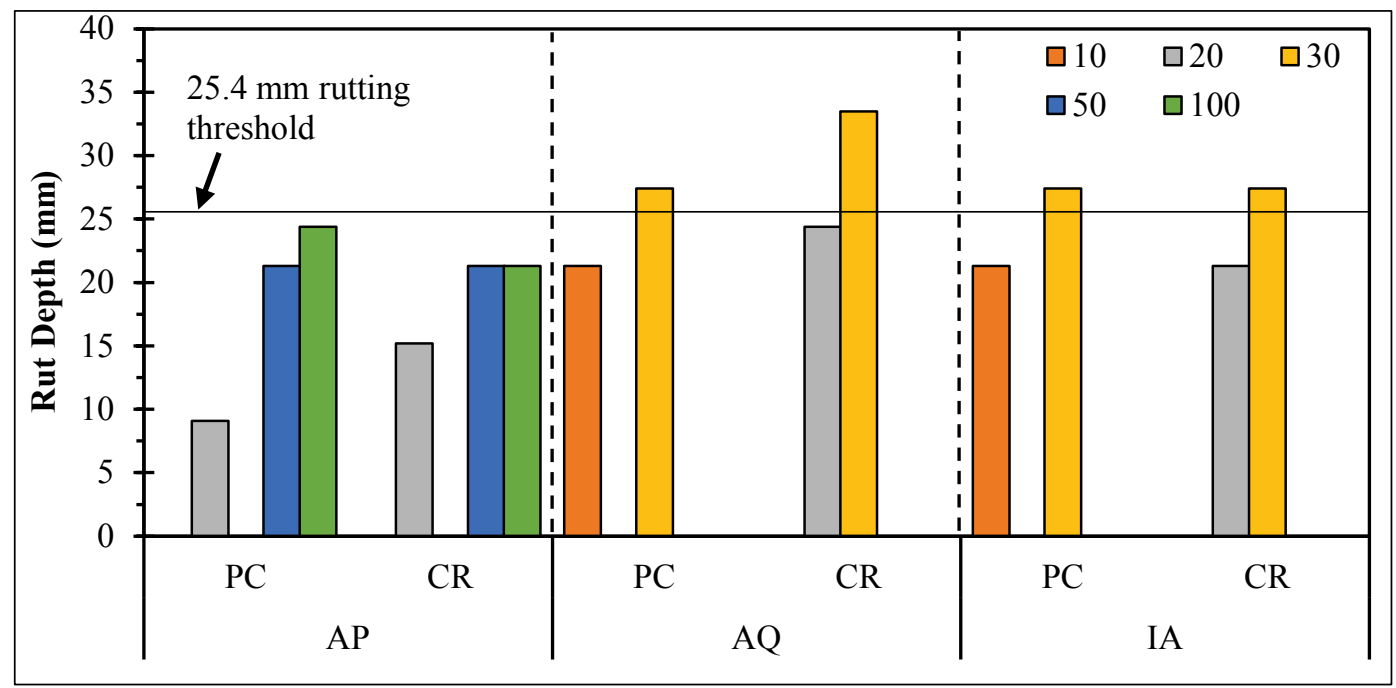

Figure 4.3 is an equality plot comparing CR and PC compaction methods where direct pairs of data were available (i.e., rut measurements for both PC and $\mathrm{CR}$ at the same pass level). Figure 4.3 was constructed using straightedge rut measurements from Figure 4.2. In general, patches compacted with the CAT roller tended to yield higher rut depths than those compacted with the plate compactor. Considerable variability is present, however. Regression through the origin illustrates that, on average, a $25.4 \mathrm{~mm}$ rut depth for PC-compacted patches was equivalent to a $26.9 \mathrm{~mm}$ rut depth for CR-compacted patches. Given the limited and variable data available, this is not a meaningful finding. Overall, rutting resistance was not meaningfully impacted by one compaction method over another.

Figure 4.3. $C R$ and $P C$ comparison using straightedge rut measurements.

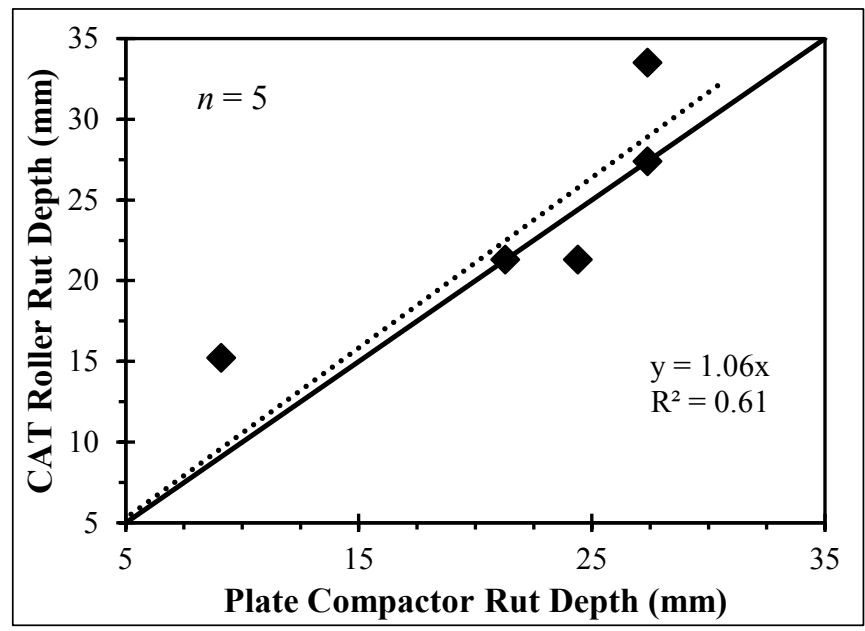


Figure 4.4 provides an equality plot comparing rut measurements obtained via surveys (rod and level) or a straightedge. Data points were either on the equality line or below it, indicating that survey and straightedge measurements were identical in some cases and, where they were not, straightedge measurements produced greater rut depths. Cases in which survey and straightedge measurements were not equal occurred when upheaval was present on either side of the wheel path. This increased the elevation of the straightedge relative to surrounding pavement since it was resting on this upheaved material when measurements were taken. Conversely, survey measurements used the surrounding pavement as the reference for rut measurements.

Figure 4.4. Comparison of survey and straightedge rut measurements.

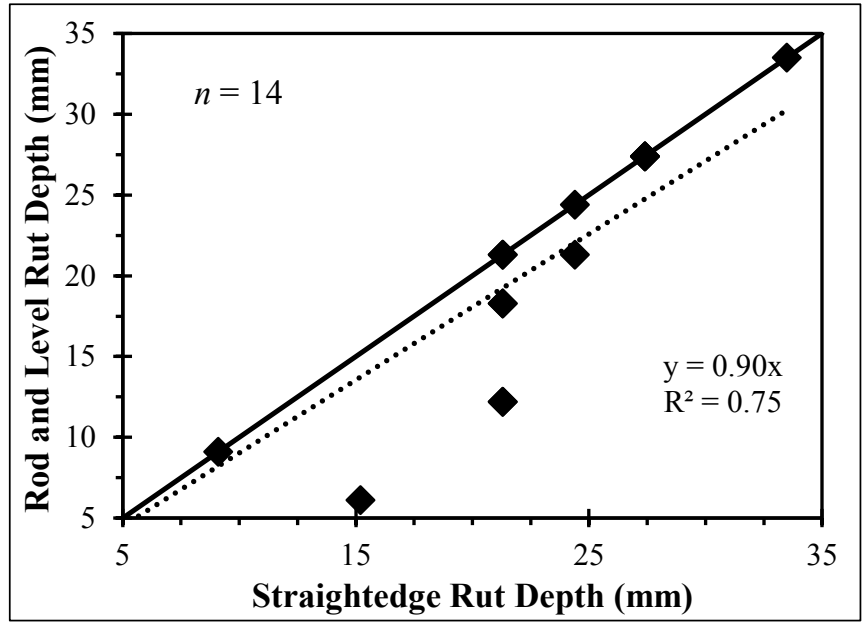




\section{Discussion of Results}

This chapter provides a joint discussion of laboratory and field results presented in Chapters 3 and 4. Discussion focuses primarily on rutting behaviors since rutting characterization was the primary focus of the project.

Laboratory rut testing of all nine cold mix products tested produced results that could be neatly grouped into one of two performance categories. Those that failed APA testing at 2,000 cycles or less (referred to as Group B products in Chapter 3) all utilized traditional cutback asphalt formulations. Note that this group included the current recommended material, IRR. Conversely, all products that withstood 8,000 cycles during APA testing (Group A products) utilized waterreactive binder formulations. This is notable given the considerable performance gap between the two groups.

Field rut testing of the current recommended material, IRR, and the three Group A cold mix products further illustrated the benefits of the waterreactive cold mix products in the context of rutting performance. Though all products were not tested at the same time due to AP unavailability in Phase 1 field testing, AQ and IA stood out from IRR with relative ease. In Phase 2 where AP was tested, AP withstood approximately three times as many load cart passes as AQ and IA. Therefore, where rutting performance is of primary interest, both laboratory and field indicators suggest waterreactive cold mix products should be considered over IRR or any other traditional cutback cold mix product.

While the water-reactive cold mix products tested ranked noticeably higher than cutback products in rutting resistance, it should be acknowledged that there are also other factors to consider. Given the temperature-dependent nature of asphalt materials, increasing performance on one end of the temperature spectrum (e.g., rutting, which is typically associated with higher temperatures) generally implies decreasing performance at the other end of the temperature spectrum (e.g., cracking and durability, which are typically associated with intermediate and low temperatures). One major distress likely associated with lower-temperature durability and potentially of concern would be FOD production. Because this project focused on 
rutting behaviors (which is one of the primary concerns with cold mix products because of the solvent and high air voids), it did not evaluate in any meaningful way other properties where issues with the water-reactive mixes could be encountered.

Durability and cracking behaviors of the water-reactive cold mixes could be an area for further study. It could be useful to compare lowertemperature performance of water-reactive cold mixes to conventional cutback cold mixes since lower-temperature properties of any cold mix product do not appear to have been studied in any great detail. It is, however, possible that the high air voids typical of cold mix products could mask any durability-related benefits, if any, of conventional cutback products. In that case, differences between lower-temperature properties of water-reactive and cutback products may not be nearly as apparent as the differences in rutting performance were in this report.

Curing time for a given product contributed considerably to observed rutting behaviors, which is a reasonable trend. Laboratory rutting data shows that rutting resistance improved slightly from 1 to 5 days of curing, with improvements tapering with cure time. This is expected and also demonstrates that more strength gain occurs early on (1 day or less) rather than later ( 5 days). Field data supports this behavior, showing significant improvement between $\mathrm{C}_{1}$ ( $2 \mathrm{hr}$ ) and $\mathrm{C}_{3}$ (3 days) (no 1-day testing was conducted in the field). Rutting resistance at $2 \mathrm{hr}$ was almost negligible in all cases, while it was adequate (met the 100-pass objective at less than $25.4 \mathrm{~mm}$ rut depth) for AQ and IA at 3 days.

$\mathrm{C} 1$ does not appear to be sufficient because, though it meets the time requirement for expedient repairs, it does not perform adequately under traffic. Conversely, a 3-day curing time may not be practical depending on the application (e.g. expedient base recovery after attack). None of the COTS cold patch products evaluated during this effort met or exceeded the threshold values of $100 \mathrm{~F}-15 \mathrm{E}$ passes at $2 \mathrm{hr}$ cure time.

It is worth noting that in extreme scenarios where minimal passes are required of a cold patch repair, such as base evacuation, $\mathrm{C} 2$ yielded considerably better rutting performance than $\mathrm{C} 1$. Both conditioning protocols required a total of $2 \mathrm{hr}$ to conduct with the only difference being that patches were also heated for $20 \mathrm{~min}$ at some point during the 2-hr period using the infrared heater. If an equivalent heating mechanism was available, it does appear that it would be advantageous to use to accelerate curing to some degree and, ultimately, obtain longer life from the patch. 


\section{Conclusions and Recommendations}

Laboratory air void, rutting, and IDT data was presented in this report alongside field rutting data with simulated $\mathrm{F}-15 \mathrm{E}$ traffic for nine cold mix asphalt repair products and four conventional HMA control mixtures. Key observations from this study are as follows:

- Cold mix $V_{a}$ levels were on the order of 2-3 times greater than typical target $V_{a}$ levels for HMA during construction,

- Water-reactive cold mix products, withstanding a full 8,000-cycle APA test, exhibited significantly more favorable rutting behaviors than traditional cutback products that withstood 2,000 cycles or less,

- Within water-reactive cold mix products, AQUA PATCH (AP) yielded the highest rut resistance in both laboratory and field testing. Aquaphalt ${ }^{\mathrm{TM}}$ 6.0 (AQ) and Instant Asphalt ${ }^{\mathrm{TM}}$ 6.0 (IA) demonstrated similar behaviors to one another,

- In field testing, rutting resistance of the HMA control mixture, CM-F, was on the order of 2-5 times greater than water-reactive cold mixes AQ and IA (the only two products directly compared to HMA),

- Trends were relatively consistent between laboratory and field rut testing in that AP ranked most favorably, followed closely by AQ and IA, and trailed considerably by cutback products. This supports other findings that the APA can be a useful tool in ranking materials on a laboratory-scale,

- Curing time meaningfully improved rutting results with effects tapering with time. Laboratory rut depths for water-reactive products decreased 35 to $52 \%$ ( $44 \%$ average) from 1 to 3 days of curing and 2 to $32 \%$ (19\% average) from 3 to 5 days. Field load cart passes to failure for water-reactive products increased from 4 to 100 from $2 \mathrm{hr}$ to 3 days of curing.

Based on findings in this study, key recommendations are as follows:

- Lower-temperature testing should be conducted, particularly for the water-reactive products, to evaluate durability and cracking properties. From a materials perspective, this is important to consider for the water-reactive products since it is possible that the increased rutting resistance comes at a cost with regard to lower-temperature durability. 
This should also be considered from a base operations perspective. Generally, cold mixes are used to provide temporary patches only. In this case, long-term durability of the patch may be of less concern than the immediate ability to apply traffic and not rut. However, FOD potential should also be considered as it is a possible byproduct of increased rutting resistance,

- Depending on lower-temperature durability as well as situational priorities, replacement of Instant Road Repair ${ }^{\circledR}$, the current recommended product, with a water-reactive product should be considered, especially if the primary interest is rutting resistance of temporary patches. Of the three water-reactive products tested, AQUA PATCH (AP) would be the most favorable product in terms of rut resistance, though Aquaphalt ${ }^{\mathrm{TM}}$ 6.o (AQ) and Instant Asphalt ${ }^{\mathrm{TM}}$ 6.0 (IA) would also be suitable (and extremely preferable relative to cutback products) options. Logistical issues such as product acquisition and distribution would also be important considerations in addition to performance. Further, the shelf life of this category of product should be studied to better understand their ability to be pre-positioned for use as a repair material,

- When possible, an HMA material should be used for patching purposes as HMA typically provides greater overall performance than cold mix. One the greatest logistical issues associated with HMA in a base operations context is whether or not HMA can be produced and transported to the base while maintaining compaction temperatures until placement of the patch. HMA produced in advance and cooled to form loose mix could be utilized if a method for rapidly heating the loose mix was available. Development of such a method may be worth further investigation. 


\section{References}

American Association of State Highway and Transportation Officials (AASHTO). 2010. Standard method of test for determining the rutting susceptibility of hot mix asphalt (HMA) using the asphalt pavement analyzer (APA). Designation T34010. Washington, DC: American Association of State Highway and Transportation Officials.

. 2013. Standard method of test for bulk specific gravity $\left(G_{m b}\right)$ and density of compacted hot mix asphalt (HMA) using automatic vacuum sealing method. Designation T331-13. Washington, DC: American Association of State Highway and Transportation Officials.

American Society for Testing and Materials (ASTM). 2003. Standard test method for theoretical maximum specific gravity and density of bituminous paving mixtures. Designation D2041-03a. West Conshohocken, PA: American Society for Testing and Materials.

. 2012. Standard test method for indirect tensile (IDT) strength of bituminous mixtures. Designation D6931-12. West Conshohocken, PA: American Society for Testing and Materials.

Mejías-Santiago, M., F. Valle-Roldán, and L. P. Priddy. 2010. Certification tests on cold patch asphalt repair materials for use in airfield pavements. ERDC/GSL TR-1014. Vicksburg, MS: U.S. Army Engineering Research and Development Center.

Rushing, J. F., M. Mejías-Santiago, and J. D. Doyle. 2013. Assessment of warm-mix asphalt for heavy traffic airfields. Transportation Research Record No. 2371. Washington, DC: Transportation Research Board. 


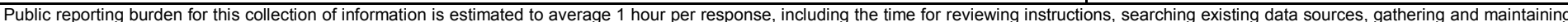

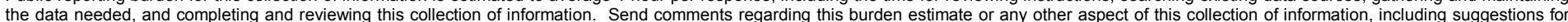

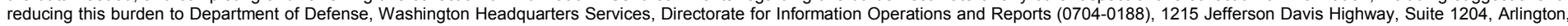

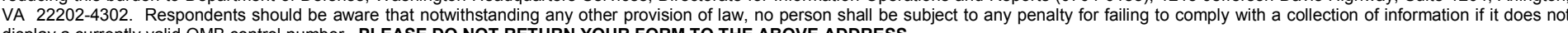
display a currently valid OMB control number. PLEASE DO NOT RETURN YOUR FORM TO THE ABOVE ADDRESS.
1. REPORT DATE (DD-MM-YYYY)
June 2017

\section{TITLE AND SUBTITLE}

Rutting Performance of Cold-Applied Asphalt Repair Materials for Airfield PavementS
3. DATES COVERED (From - To)

5a. CONTRACT NUMBER

5b. GRANT NUMBER

5c. PROGRAM ELEMENT NUMBER

5d. PROJECT NUMBER 448464

Ben C. Cox, John F. Rushing, and Web Floyd 5f. WORK UNIT NUMBER

8. PERFORMING ORGANIZATION REPORT NUMBER

ERDC/GSL TR-17-10

Geotechnical and Structures Laboratory

U.S. Army Engineer Research and Development Center

3909 Halls Ferry Road

Vicksburg, MS 39180-6199

\section{SPONSORING / MONITORING AGENCY NAME(S) AND ADDRESS(ES)}

Headquarters, Air Force Civil Engineer Center

139 Barnes Avenue, Suite 1

Tyndall AFB, FL 32403-5319

10. SPONSOR/MONITOR'S ACRONYM(S)

HQ-AFCEC

11. SPONSOR/MONITOR'S REPORT NUMBER(S)

\section{DISTRIBUTION / AVAILABILITY STATEMENT}

Approved for public release; distribution is unlimited.

\section{SUPPLEMENTARY NOTES}

\section{ABSTRACT}

Cold-applied asphalt mixtures are often used for pavement repair, primarily because of the small quantities involved and/or the unavailability of hot-mixed asphalt. These cold-applied mixtures have poorer rut-ting resistance than hot mix asphalt because additives, often solvent, are required to provide adequate workability for them to be placed at ambient temperatures. This study primarily evaluated the rutting performance of nine commercial cold mix asphalt repair materials. Both laboratory wheel tracking data and field rutting performance were used in the evaluation with the focus on identifying suitable materials for re-pairing airfield pavements subjected to high-tire-pressure aircraft traffic. The commercial products included traditional cutback cold mixes as well as wateractivated repair materials. Results from both laboratory and field rutting measurements showed that the water-activated materials outperform the products containing solvent. However, none of the commercial products achieved the target threshold of less than 1 in. rut-ting after 100 passes of simulated F-15E aircraft traffic. The laboratory wheel-tracking measurements proved to be a good indicator of field performance.

\begin{tabular}{|ll|}
\hline 15. SUBJECT TERMS & Pavement repair \\
Cold mix asphalt & Patching material \\
Rutting & Pot hole repair \\
\hline
\end{tabular}

16. SECURITY CLASSIFICATION OF:

\section{a. REPORT}

Unclassified

\section{b. ABSTRACT}

Unclassified c. THIS PAGE

Unclassified
Pavements, Asphalt

Runways (Aeronautics) - Maintenance and repair Pavements, Asphalt - Live loads

\begin{tabular}{l|c}
$\begin{array}{l}\text { 17. LIMITATION } \\
\text { OF ABSTRACT }\end{array}$ & $\begin{array}{c}\text { 18. NUMBER } \\
\text { OF PAGES }\end{array}$ \\
& 38 \\
\hline
\end{tabular}

19a. NAME OF RESPONSIBLE PERSON

19b. TELEPHONE NUMBER (include area code) 